University of Nebraska - Lincoln

DigitalCommons@University of Nebraska - Lincoln

Civil and Environmental Engineering Faculty

Publications

Civil and Environmental Engineering

3-2019

\title{
Advanced Selection Methodologies for DNAzymes in Sensing and Healthcare Applications
}

Sandeep Kumar

Shikha Jain

Neeraj Dilbaghi

Amrik Singh Ahluwalia

Ashraf Aly Hassan

See next page for additional authors

Follow this and additional works at: https://digitalcommons.unl.edu/civilengfacpub

Part of the Biochemistry Commons, Biological Engineering Commons, and the Molecular, Cellular, and Tissue Engineering Commons

This Article is brought to you for free and open access by the Civil and Environmental Engineering at DigitalCommons@University of Nebraska - Lincoln. It has been accepted for inclusion in Civil and Environmental Engineering Faculty Publications by an authorized administrator of DigitalCommons@University of Nebraska Lincoln. 
Authors

Sandeep Kumar, Shikha Jain, Neeraj Dilbaghi, Amrik Singh Ahluwalia, Ashraf Aly Hassan, and Ki-Hyun Kim 


\title{
Advanced Selection Methodologies for DNAzymes in Sensing and Healthcare Applications
}

\author{
Sandeep Kumar, ${ }^{1,3}$ Shikha Jain, ${ }^{1}$ Neeraj Dilbaghi, ${ }^{1}$ \\ Amrik Singh Ahluwalia, ${ }^{2}$ Ashraf Aly Hassan, ${ }^{3}$ \\ and Ki-Hyun Kim ${ }^{4}$ \\ 1 Department of Bio and Nano Technology, Guru Jambheshwar University \\ of Science and Technology, Hisar-Haryana, 125001, India \\ 2 Department of Botany, Panjab University, Chandigarh, 160014, India \\ 3 Department of Civil Engineering, College of Engineering, University of \\ Nebraska-Lincoln, PO Box 886105, Lincoln, NE 68588-6105, USA \\ 4 Department of Civil and Environmental Engineering, Hanyang University, \\ 222 Wangsimni-Ro, Seoul 04763, Republic of Korea \\ Correspondence — ksandeep36@yahoo.com (S. Kumar) \& kkim61@hanyang.ac.kr (K.-H. Kim).
}

\section{Highlights}

- Techniques for the selection of DNAzymes via SELEX approaches include microfluidics, nanoselection, hi-fidelity, isogenic, and automated.

- DNAzymes are able to catalyze chemical reactions with high activity and selectivity.

- DNAzymes have applications in sensing, catalysis, and healthcare.

- DNAzyme-based biosensors typically use RNA-cleavage and G-quadruplex DNAzymes.

- Suppression of cancer-related genes is an important target of DNAzymes.

- DNAzymes may be used as theranostic agents with dual utility in diagnosis and treatment.

Published in Trends in Biochemical Sciences, Vol. 44, No. 3 (March 2019), pp 190-213. doi:10.1016/j.tibs.2018.11.001

Copyright (c) 2018 Elsevier Ltd. Used by permission. 


\begin{abstract}
DNAzymes have been widely explored owing to their excellent catalytic activity in a broad range of applications, notably in sensing and biomedical devices. These newly discovered applications have built high hopes for designing novel catalytic DNAzymes. However, the selection of efficient DNAzymes is a challenging process but one that is of crucial importance. Initially, systemic evolution of ligands by exponential enrichment (SELEX) was a labor-intensive and time-consuming process, but recent advances have accelerated the automated generation of DNAzyme molecules. This review summarizes recent advances in SELEX that improve the affinity and specificity of DNAzymes. The thriving generation of new DNAzymes is expected to open the door to several healthcare applications. Therefore, a significant portion of this review is dedicated to various biological applications of DNAzymes, such as sensing, therapeutics, and nanodevices. In addition, discussion is further extended to the barriers encountered for the real-life application of these DNAzymes to provide a foundation for future research.
\end{abstract}

\title{
Glossary
}

Chromatography: a technique used for the physical separation of mixtures into their components. All forms of chromatography work on the same principle, in that, all have a stationary phase and a mobile phase. The approach is wellknown for its high sensitivity because of competitive interaction between the desired molecule and target immobilized stationary phase that are mainly composed of agarose beads.

Combinatorial chemistry: synthetic method used to prepare a library of structurally related analogues in a single process. Currently, vast ranges of simulation software are used for generating combinatorial library.

Electrochemical detection: the basic principle of electrochemical biosensors involves immobilization of selected functional DNA molecules on an electrode surface that specifically bind to a ligand, which can further lead to a change in the current or voltage.

Electrophoresis: analysis technique used to efficiently separate charged particles from each another based on differences in their mobility. It can be used to separate a variety of small and large target molecules. The major shortcoming associated is that the target molecule should have a sufficient size to induce mobility changes.

G-quadruplex DNAzyme: single-stranded G-rich aptamer sequences spontaneously form an intramolecular four-stranded helical structure (G4). Associates with the hemin group to form the catalytic G4-hemin-DNAzyme complex. This complex DNAzyme catalyzes the oxidation reaction of hydrogen peroxide to result in the generation of chemiluminescence.

Logic gate: a logic gate performs a logical operation (Boolean algebra) on given inputs in order to generate a single electrical output. Different logic gates are combined using combinatorial or sequential approaches for computer programming. Basic logic gate series include-AND, OR, NOT, XOR, NOR, NAND, and XNOR. 
Metallozymes: enzymes that require metal ions as cofactors for their catalytic activity. Although the metal cannot be bound in a permanent way to the enzyme, it is bound firmly to a specific region of the native conformation of the protein.

Michaelis-Menten kinetics: it is a fundamental equation for enzyme kinetics that relates rate of reactions [V], to substrate concentration [S] i.e., V = Kcat [Enzyme] $[\mathrm{S}] /(\mathrm{Km}+[\mathrm{S}])$. PCR: polymerase chain reaction is a molecular technique used to exponentially amplify segments of a particular DNA sequence into millions or billions of copies. The strategy involves incorporation of a PCR primer and DNA polymerase with a template strand. The method has a major limitation in that it may give false positive results.

RNA interference (RNAi): RNA molecule based regulatory system. Directs enzyme complexes to degrade mRNA molecules. Different categories of these molecules include miRNA, small interfering RNA (siRNA), and small hairpin RNA (shRNA). Secondary conformations: 2D or 3D structures attained by single-stranded nucleic acid through the use of hydrogen bonds. Stem loops, hairpins, tetraloops, and pseudo-knots are the most well-known conformations in the context of catalytic molecules.

SELEX: SELEX also known as 'selected and amplified binding site' (SAAB) and 'cyclic amplification and selection of targets' (CASTing) is an 'in vitro selection' method used for the isolation of DNA based catalytic molecules. It is an iterative method that includes selection, separation, and amplification of desired molecules.

Theranostics: is an emerging field that deals with both imaging and therapeutics in one step. Initially, it was used only for nanomaterials as they are widely explored as both imaging agents and pharmaceutical agents, but now this has been expanded to other novel materials.

XNAzymes: oligonucleotide probes with altered sugar backbones have been discovered and named xenonucleic acids (XNAs). These XNA molecules were synthesized directly from DNA templates but replicated by means of specially engineered mutant polymerases. These polymerases introduced errors, thereby producing novel XNA sequences catalyzing reactions termed as XNAzymes.

\section{DNAzymes - Catalytically Active DNA Molecules}

DNAzymes are functionally active, synthetic single-stranded (ss) DNA molecules that are capable of catalyzing biological reactions similarly to RNAzymes and proteins. DNA molecules as enzymatic molecules are synthesized using the in vitro selection method known as systemic evolution of ligands by exponential enrichment (SELEX; see Glossary). Selected DNAzymes consist of a central catalytic domain flanked by two sequences specifically designed to bind to the desired target sequence. These molecules, upon binding to the complementary sequence, can catalyze the cleavage of the target sequence using the 
catalytic domain. DNAzyme-based molecular catalysts generally depend on a metal ion cofactor for catalysis. It has also been reported that DNAzyme-mediated catalysis follows Michaelis- Menten kinetics and can enhance the rate of reaction up to many folds [1]. Unlike proteins and RNAzymes, there are no reports of naturally occurring DNAzymes.

The invention of self-splicing RNA catalysts raised the possibility that DNA molecules might also be used as catalysts. Catalytic DNA molecules are not as efficient as protein molecules because of the inert nature of the monomeric units and the lower diversity of functional groups. Nonetheless, they are reported to have the advantage that they can form diverse structures and their easy manipulation has promoted their use as potent catalytic molecules. The development of an in vitro selection technique which enabled the screening of functionally improved synthetic catalytic biomolecules brought about a revolution in this field. These biomolecules are screened from a chemically synthesized pool of oligonucleotides generated through combinatorial chemistry $[2,3]$.

After many research efforts, Breaker and Joyce [4] successfully invented the first DNAzyme. Eventually, DNAzyme-based research gained momentum, stimulating the synthesis of a variety of DNA molecules that target a range of molecules [5]. Since then, numerous DNAzymes have been developed that catalyze a broad array of reactions, demonstrating them to be true biocatalysts alongside ribozymes and proteinaceous enzymes, as shown in Figure $\mathbf{1}$.

In addition to the synthesis and mechanism of reaction catalyzed by DNAzymes, their significance in various healthcare applications has been increasingly emphasized. However, this field has not yet been critically reviewed. To date, there are only a few reports that have addressed the synthesis and applications of DNAzymes. Earlier reviews have focused on DNAzymes as biosensors, DNAzyme-nanoparticle (NP) conjugates, and DNA machines [6-8]. In this review we aim to combine all the important features related to the design of DNAzymes, the possible chemical reactions that can be catalyzed, and the exploration of current and future applications in healthcare. In the first part, we summarize the methodologies used for the synthesis of DNAzymes. In the next section, we discuss the different types of reactions that DNAzymes can catalyze, enabling selection of the best 


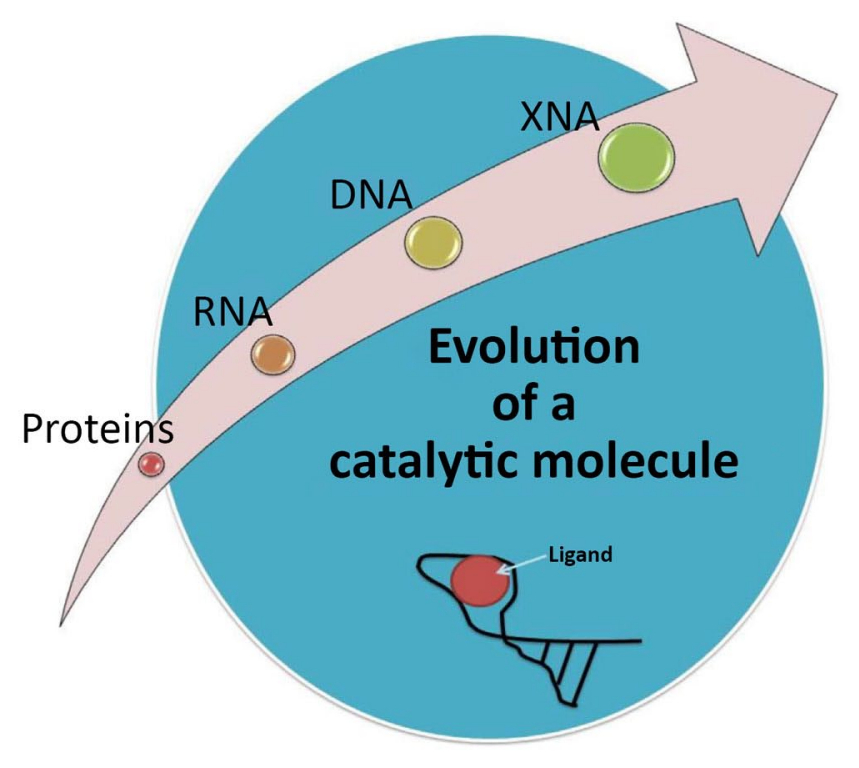

(A)

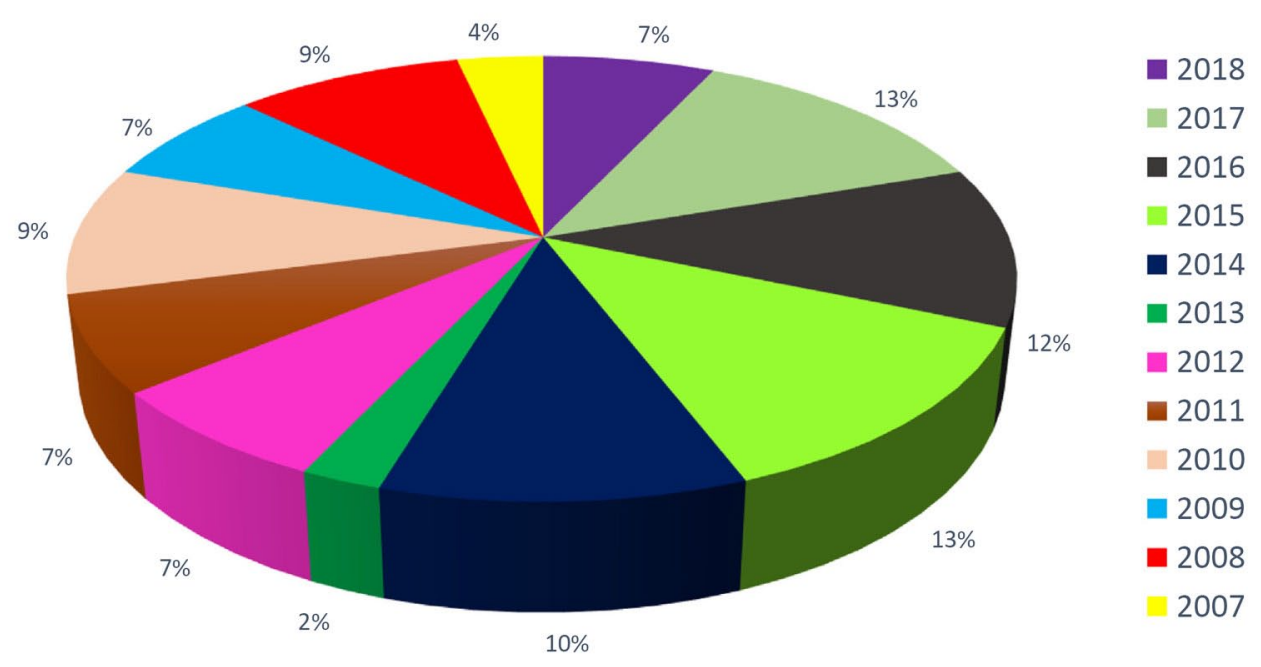

(B)

Figure 1. Evolutionary Infographics and Pie Chart Analysis of DNAzyme. (A) Schematic of the evolutionary lineage of a catalytic molecule. (B) Pie chart showing the percentage of published reviews in past decade featuring DNAzymes from the literature database (PubMed). About 86 reviews were found using keywords 'DNAzyme', 'DNA catalysis', and 'applications'. Abbreviation: XNA, xenonucleic acid. 
DNAzyme for each of the desired applications. Finally, we highlight important applications of DNAzymes in sensing, therapeutics, molecular devices, and nanodevices. In addition, barriers and future prospects of DNAzymes are also discussed to aid future research efforts in this field. Overall, this review aims to provide a precise and clear view of DNAzymes, ranging from basic concepts to practical applications.

\section{Molecular Evolution of a DNA Molecule: A New-Age Catalytic Molecule}

DNA and RNA are naturally present as molecules of heredity but also have integral properties as catalysts. The most crucial feature of an enzymatic molecule is the positioning of functional groups to generate diverse 3D conformations. DNA duplexes have limited structural diversity because of their constrained 3D conformation, making double-stranded (ds) molecules less efficient at catalyzing biochemical reactions. Moreover, the stability and the absence of 2 ' hydroxyl from the sugar moiety of a DNA molecule make the dsDNA molecule less efficient when catalyzing a reaction. Therefore, ssDNA molecules have been explored as catalytic molecules [5].

A crucial feature of ss nucleic acids is that they can form composite 2D or 3D structures by employing interdomain contacts. The generation of a variety of stabilized secondary structure conformations by ssDNA molecules has been widely demonstrated [9]. Researchers have mainly focused on the development of new functional DNA molecules (aptamers/DNAzymes) with the help of combinatorial chemistry. However, evolution of functionally active biopolymers from conventional polymers is not an easy task because their configuration imposes significant hindrances, such as geometrical arrangement, stability, and functional complexity [10]. Unlike protein-based enzymes, DNAzymes can be easily synthesized using in vitro selection [6]. This is mainly because of the small $4 \mathrm{n}$ sequence space and the degeneracy of codons. Comparatively, protein molecules have a large sequence space of 20 amino acids. Moreover, most common protein secondary structures such as a-helices and b-sheets are unstable if they fail to adopt tertiary conformations due to the inherent cooperative nature of folding domains. By contrast, the secondary conformations adopted by ss nucleic acid molecules (e.g., hairpins and stem-loops) are relatively 
stable. Another important factor is the availability of biochemical tools, such as DNA polymerases, reverse transcriptases, and RNA polymerases that facilitate the synthesis and design of new DNAzymes. These molecular tools are readily available for nucleic acid catalysts, but not for the selection of synthetic protein sequences.

In addition, DNAzymes are also favored as catalysts over RNAzymes due to their in vivo stability [11]. The presence of a carbonhydrogen $(\mathrm{C}-\mathrm{H})$ bond at the $2^{\prime}$ position of the sugar moieties of DNAzymes confers increased stability and reduced reactivity. DNAzymes are also less expensive than RNAzymes and can be easily synthesized because there is no need for elaborate steps such as reverse transcription. These factors favor the in vivo performance and implementation of DNAzyme for various healthcare applications. The merits and demerits of different catalytic molecules are described in Table 1. Moreover, DNA-based enzymes can further contribute to advances in nucleic acid chemistry, especially through the synthesis of artificial enzymes. Synthetic genetic polymers, known as xenonucleic acid (XNA) with ameliorated stability, can fold into a definite specific conformation capable of forming the catalytic unit 'XNAzyme' [12]. Nonetheless, it should be noted that DNA enzymes are now active areas of research that are being investigated for technological applications.

Table 1. Comparison of Merits and Demerits between Protein Enzymes, RNAzymes, and DNAzymes to Evaluate the Best Catalytic Molecule

\begin{tabular}{|c|c|c|c|}
\hline Order & Catalytic molecule & Merits & Demerits \\
\hline 1 & Proteins & $\begin{array}{l}\text { Stabilized transition state } \\
\text { Diversity of functional groups } \\
\text { Very high rate of reaction }\end{array}$ & $\begin{array}{l}\text { Require coenzyme for catalysis } \\
\text { Unstable secondary structure } \\
\text { Random sequence generation is } \\
\text { troublesome } \\
\text { Large size }\end{array}$ \\
\hline 2 & RNAzymes & $\begin{array}{l}\text { New catalytic molecule through } \\
\text { in vitro selection } \\
\text { Possible secondary and tertiary } \\
\text { conformations } \\
\text { Chemical and enzymatic stability } \\
\text { Minor batch variation }\end{array}$ & $\begin{array}{l}\text { In vivo stability } \\
\text { More complex design owing } \\
\text { to reverse transcription step }\end{array}$ \\
\hline 3 & DNAzymes & $\begin{array}{l}\text { Chemically more stable } \\
\text { Inexpensive } \\
\text { Easy synthesis } \\
\text { Easy to modify for systemic delivery }\end{array}$ & $\begin{array}{l}\text { Research is still in progress } \\
\text { Limited structural information }\end{array}$ \\
\hline
\end{tabular}




\section{A Combinatorial Selection Method, SELEX, To Synthesize New Catalytic DNA}

Combinatorial chemistry is a novel method to construct libraries that contain an astronomically large number of different but structurally similar molecules. Progress in this field has led researchers to employ combinatorial chemistry for the synthesis of a large number of unique and rare compounds of industrial significance [6]. Current challenges, such as the synthesis of catalytic molecules using genetic elements, connect two promising fields of modern science (i.e., combinatorial chemistry and molecular biology). SELEX is a combinatorial chemistrybased in vitro selection method used for the synthesis of DNAzymes. The fundamental concept behind this approach is that the desired molecule may be found among a given pool of arbitrary sequence molecules, but identifying and isolating these rare desired molecules is challenging. The basic protocol of SELEX starts with a large pool of random sequences, followed by a multiple selection scheme involving separation methodologies and the amplification of selected sequences (Figure 2).

Designing a good combinatorial library is a key step in the selection of specific catalytic molecules (Box 1 overviews factors that influence the selection of DNAzymes). The library of optimized molecules is then incubated with the ligand of interest, followed by elution of bound oligonucleotides. Pools enriched in the molecules of interest are then further separated using a range of partitioning methods.

Different types of separation methodologies such as (i) filtration, (ii) electrophoresis, (iii) chromatography, and (iv) magnetic separation were exploited to separate target specific aptamers from optimized libraries. Among these separation techniques, chromatography has been used most often for the selection process because it can offer high selectivity and specificity [13]. The selection and purification procedure is then repeated multiple times, and the numbers of selected aptamers are reduced with each cycle of selection. Hence, conventional SELEX requires a timeframe of months for the generation of the desired final molecule [14].

After binding and separation, selected molecules are amplified, and PCR is used for the amplification of DNA and RNA [5]. SELEX is thus a repetitive process that includes binding, separation, and the 
$\mathbf{5}^{\prime} \quad \mathbf{N}_{20-60} \quad 3^{\prime}$

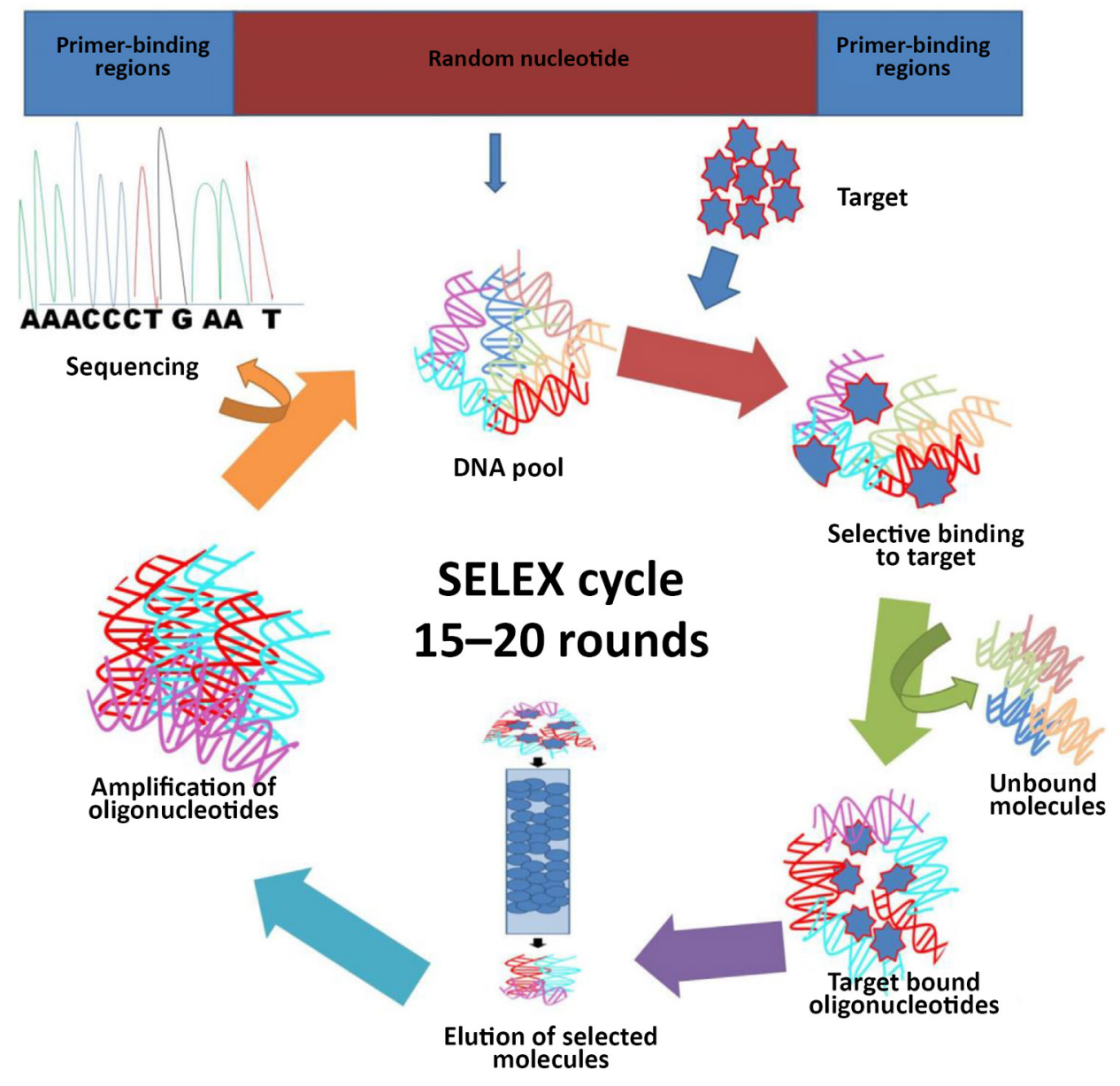

Figure 2. Schematic Demonstration of the Selection of DNAzyme/Aptamer Molecules Using the Conventional SELEX (Systemic Evolution of Ligands by Exponential Enrichment) Process. The selection process involves multiple steps such as (i) pool of ss DNA incubated with target molecules for selective binding, (ii) elution of bound sequences through different separation techniques, (iii) amplification of eluted sequences, and (iv) analyzing the best sequences by sequencing after 15-20 rounds.

amplification of selected molecules. Optimally, 15-20 rounds of selection and amplification have been suggested for the initial identification of target molecules; however, the reliability of single rounds as an alternative to conventional SELEX has been demonstrated by many research groups [14]. In addition, advances in substituent methods have recently gained momentum for the selection of desired molecules [15-17]. Refinement of the strategy has focused on crucial steps in the 
Box 1. Key Factors Influencing the Design and Synthesis of DNAzymes

Nucleotide Sequence

A 20-60 nt random sequence is thought to be optimal for the synthesis of DNA aptamers [117]. Although longer sequences allow more complex structures to be generated, they can form self-aggregates, resulting in precipitation upon prolonged incubation. By contrast, smaller nucleotide sequences have less likelihood of generating the desired sequences owing to limited structural diversity. Short oligonucleotide pools can be generated using the basic phosphoramidite chemical method, which has advanced further with automated solid-phase DNA synthesis machines [118]. The synthesis of much larger oligonucleotides (>100 nt) is a more challenging process. The most appropriate techniques are based on the incorporation of precise mutations into cloned sequences or on enzymatic synthesis (ligation-based synthesis and PCR-based approaches) [119]. More advanced technologies based on microarray systems (e.g., photolithography, microfluidics, and microchips) [120] can also be used for oligonucleotide synthesis. These new methodologies can not only lead to a reduction in cost and time but also generate longer sequences.

\section{Secondary Structure}

The degree of secondary/tertiary structure is another important factor because it is related to the generation of the binding site [121], and high-affinity aptamers can require a more complex structure. Computation-based aptamer design has been proposed to include secondary complexity [122].

\section{Catalytic Junction}

ApivotalaspectinthedesignofDNAzymesistheselectionofthecleavage junctionbecausethiscanaffectthefolding,cleavagerate, binding affinity, and, most importantly, the probability of producing an effective 8-17 DNAzyme motif $[121,123]$.

\section{Fixed Flanking Sequences}

Specific structural sequences flanking random regions, such as primer-binding sequences and RNA domains, have been employed for the amplification of selected oligonucleotide sequences [124]. The addition of an RNA domain within the random DNA sequence increases the likelihood of occurrence of the desired catalytic molecule. There are several factors that must be considered when designing and optimizing 
these constant regions, such as the formation of primer dimers, secondary structure formation, length, and GC content.

Target Concentration

An optimal target concentration is used to avoid nonspecific binding and to improve the efficiency of the SELEX selection procedure. Various strategies have been proposed and successfully exploited, including (i) addition of spacer molecules that can change the length of aptamers, (ii) coupling with a fluorescent molecule, and (iii) incorporation of unnatural bases that can help to increase the diversity of catalytic molecules $[80-82,124,125]$. The impact of optimal target concentration and nonspecific binding has been assessed in relation to the efficiency of the selection process [126].

Underestimating anyone of these parameters may lead to undesirable results. Therefore, it is very important to establish an optimal value for the above-mentioned factors during the iterative process to ensure that quality results are obtained.

conventional SELEX process, including different methods of immobilization, separation, removal of unwanted aptamer molecules, quantification, binding efficiency, real-time monitoring, and many more.

\section{Current Emerging Concepts of the SELEX Technique}

For a long time researchers considered prototypical methods such as negative, counter, blended, and toggle SELEX (Table S1 in the supplementary information online) as efficient techniques for the selection of DNAzymes. These classical methods are still the basis of high-throughput screening processes. Most current SELEX applications utilize multiple methods that are suitable for diverse classes (Figure 3). Major forms of SELEX which have recently gained momentum are acclimatizing techniques such as microarrays, sequencing, and microfluidics. 


\section{SELEX timeline}

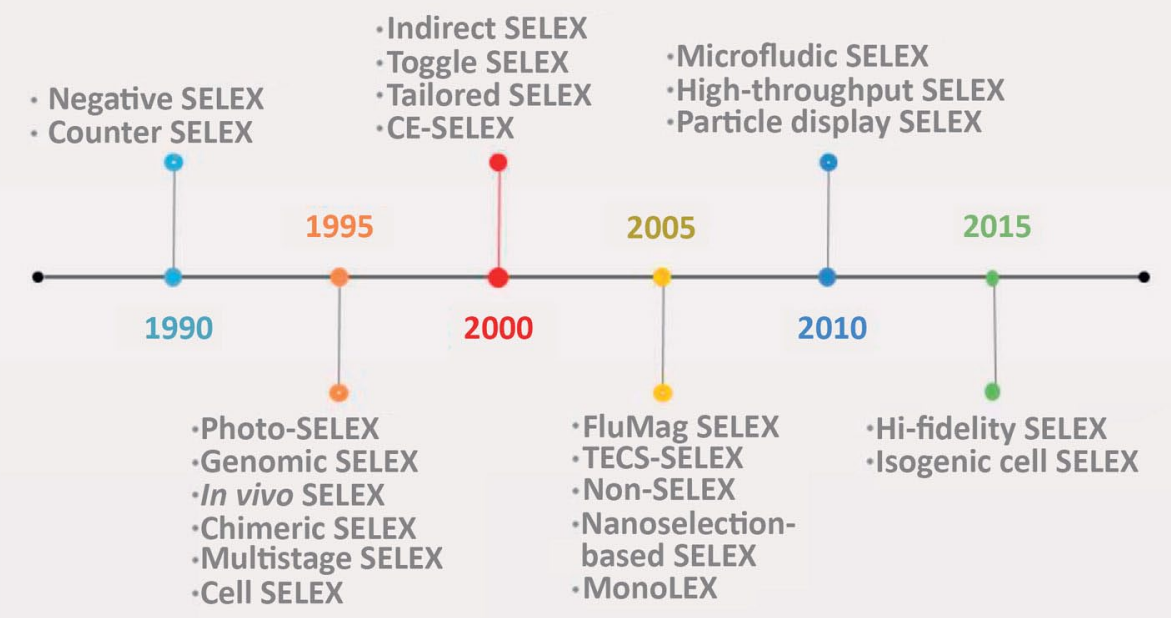

Figure 3. Historical Evolution of Key SELEX Processes. This scheme shows major SELEX methodologies from their discovery (i.e., Negative SELEX) to present advancements (i.e., Hi-fidelity SELEX).

\section{Cell-SELEX}

The original SELEX method utilizes purified proteins as targets for the selection of high-affinity aptamers. An important issue related to these aptamers was their low performance in clinical trials. The reason is the inability of aptamers to bind native proteins because they adopt a different conformation in vivo. In view of such findings, a new concept of a complex native molecule as a SELEX target came into existence. Later, it was applied to further upgrade the methodology for selection against whole cells [18]. However, a major challenge arises while performing cell-SELEX of aptamers against multiple targets, and counterselection is essential to avoid nonspecific binding. The correct choice of target cell and control cell is also an important factor to be considered while carrying out cell-SELEX. The cell-SELEX process does not require elucidation of the molecular markers on the target cell surface, which further simplifies the in vitro selection method. Indeed, the whole SELEX process is a basic, rapid, reproducible, and 
direct approach to generate high-affinity aptamers in the nanomolar-to-picomolar range [19]. Cell-SELEX is hence an attractive strategy for implementation in diagnostic applications.

A major concern in terms of modern healthcare is to devise and implement therapeutics for cancer. Cell-SELEX can be utilized for the enrichment and identification of aptamers targeting cancer cells [1820], and the technique can be extended to diverse targets including stem cells, virus-infected cells, and bacterial cells [21-23]. It can also be used to identify new biomarkers and drug targets for the treatment of major diseases [24].

Cell-SELEX holds high potential in healthcare applications, but there are some limitations that constrain the affinity of the aptamer molecules identified. For example, the presence of dead cells in the target preparation can adversely affect the selection procedure and can result in nonspecific DNA molecules. In addition, (i) difficulties in the identification of targets due to the complex nature of the cell membrane, (ii) reduced binding affinity because of the repulsive force between a negatively charged DNA molecule and similarly charged cellmembrane components, and (iii) separation of the binding complex is not efficient because the target cells are not fixed [20]. However, numerous efforts have been made by researchers to overcome these limitations and to further optimize the technology. Optimization of the methodology contributed to the development of a more efficient version of cell-SELEX that offers a glimpse of fast and current research. Raddatz et al. eliminated dead cells using fluorescence-activated cell sorting (FACS), and named the method FACS-SELEX [25]. In comparison to other existing methods, this technique requires less time to complete, mainly due to the synchronous execution of both positiveand negative-selection steps. Cell- internalization SELEX [26] is capable of selecting aptamers that can deliver drugs directly into the cytoplasm of the target cells. Aptamers slow in internalization were eliminated using stringent washing. The major issue with this method is the choice of target cells (i.e., a cell line over- expressing the target protein) and a comparable negative cell line. Evolution of the internalization methodology increased the efficiency of cell-SELEX, although it remains a cumbersome procedure. 3D cell SELEX [27] was recently developed based on a 3D cell culture, employing magnetic levitation technology, that mimics the tissue microenvironment under 
in vitro conditions. These studies suggest that the technology is improving day by day to promote the widespread use of SELEX in healthcare applications.

\section{In Vivo SELEX}

Although the SELEX approach has been employed to enrich for aptamers targeting either purified substrate or cells expressing the target of interest, the conditions employed did not accurately reiterate the in vivo configuration and stability. As a result, therapeutic aptamers did not perform ideally under in vivo conditions. The in vivo stability of aptamers is of utmost importance in the context of therapeutics, and in vivo SELEX methodology has been demonstrated to select highly stable and active aptamers within the living cell environment [28]. This approach has many advantages for clinical research, and many research groups have used this technology to select ligands targeting different diseases, especially tumors [29] as they can selectively localize and inhibit diseased tissue while helping to decrease the level of pharmacologic optimization.

Another important task in therapeutics is to discover drugs that can penetrate the blood-brain barrier (BBB). In vivo SELEX has been used in conjunction with artificial BBB cell culture to select aptamers that can be delivered to the brain $[30,31]$. However, more rounds of selection are necessary to obtain the desired aptamers, making the system more complex. Further improvements can be achieved by using a smaller library to reduce the number of rounds of selection that are required, or by conjugation to NPs to improve delivery [32]. The combination of in vitro and in vivo SELEX can be more appropriate for designing aptamers. This approach enabled the real-time detection of aptamer-binding targets in live cells, and has potential in many healthcare applications [33].

\section{Toggle SELEX}

The toggle SELEX method follows the conventional strategy but employs multiple targets. Rotation of targets during screening allows the selection of ligands for conserved domains of related targets, and has facilitated the selection of catalytic molecules for healthcare applications $[22,23]$ where crossreactivity may enhance in vivo viability because preclinical evaluation of therapeutic molecules sometimes 
fails due to low in vivo efficacy. Since, clinical trials require crossreactivity to diagnose multiple targets toggle SELEX can be extremely helpful for drug discovery.

To exploit the toggle strategy in the selection of ligands that favors crossreactivity, the target should be properly selected. A larger surface area leads to the selection of sequences against various sites of a target, some with lower affinity and others with higher affinity, while targeting individual epitopes often results in a weakly interacting molecule. Toggle SELEX was applied for the generation of aptamers that have broad specificity for different clinical isolates of influenza virus [22]. Very recently, in light of the potential benefits of toggle SELEX, it has been used for the selection of an aptamer that can target different Gram-positive and Gram-negative bacteria [23].

\section{High-Throughput SELEX}

For high-throughput screening of combinatorial libraries, kinetic capillary electrophoresis-based methods, such as nonequilibrium capillary electrophoresis of equilibrium mixtures (NECEEM), equilibrium capillary electrophoresis of equilibrium mixtures (ECEEM), and sweeping capillary electrophoresis (SweepCE), have been employed for the selection of catalytic molecules [34]. These methods were primarily based on the separation of a mixture containing an oligonucleotide and the target using gel-free capillary electrophoresis. To further increase the efficiency of CE-SELEX, a fraction collection approach has been integrated into a newer version named FCE-SELEX. The most striking feature of FCE-SELEX is its ability to select aptamers in a single round of selection [35].

A particle display bead-based method has also been used for highthroughput selection of high-affinity aptamers [36]. In this method, instead of immobilizing target molecules, the aptamers are used as the stationary phase. For this purpose, each aptamer is converted to aptamer particles using emulsion PCR. Subsequently, these aptamer particles are selected against a fluorescent-labeled target and screened by FACS.

These high-throughput selection methods are currently widely used to produce aptamers against different molecules $[34,37,38]$ and have been suggested as a highly rapid and economical method for the generation of catalytic molecules. 


\section{Microfluidic SELEX}

Microfluidics is a discipline of science that deals with the use of fluids at the microlevel to achieve automated and high-throughput point-of-care devices. In microfluidics SELEX, micro-volumes of targetDNA mixtures are passed through microfabricated channels and further separated using a high-voltage current [39]. This technique can be operated continuously for a long time to isolate conjugates with low mobility and with low dilution of samples. It has been assumed that reducing the number of target molecules may lead to the identification of aptamers with higher affinity. In addition, the short transit time enhances the applicability of the method, and this microsystem provides a rapid and effective screening protocol for aptamers with potential diagnostic applications [40-42].

\section{Automated SELEX}

Automation can ensure superior reproducibility, and several different molecular methods (e. g., sequencing, PCR, and microarrays) have already been automated. Consequently, this resulted in ease of techniques by simply reducing the time needed for manipulation and arrangements. Because SELEX is a very time-consuming and repetitive process, automated versions were developed and used to select catalytic molecules against different types of targets [43]. A major limitation is that, so far, automated SELEX can only be used for a single target. Hence, there is a need for an approach to multiplex target. Recently, an automated microcolumn-based 96-well plate device, MEDUSA, was developed that can process aptamer selection for multiple targets $[44,45]$. An improved version of high-throughput SELEX compatible with automation was also demonstrated by Gopinathan et al. [46]. However, fully automated frameworks require consistent integration of processes and design (e.g., software development, fabrication of instruments, and installation), and their operation requires significant expenditure and maintenance.

Semi-automated SELEX may have advantages in the selection of high-specificity aptamers. To maintain versatility, the initial selection steps are performed manually, but a robotic mechanism is employed for subsequent steps such as PCR amplification, ultrafiltration, and fluorescence detection. Many semi-automated workstations for the SELEX process have been reported. Hunniger et al. recently developed 
a semi-automated SELEX [47] platform in which the first few steps of SELEX, including incubation and elution of aptamer sequences, are carried out by an automated deep-well plate 'FISHing' procedure. These eluates were then amplified using solid phase-emulsion PCR in a process known as 'BEAMing'. This allowed simultaneous selection of aptamers against a variety of targets. A rapid automated approach that is less costly is chip-based microfluidic SELEX which permits miniaturization of the SELEX system [39]. Although chip-based automation is much more accessible, further developments of related technologies are needed.

\section{Nanoselection-Based SELEX}

Nanotechnology is currently widely applied in a variety of fields, including healthcare, materials science, and molecular biology, because it offers several advantages such as small size and large surface area, optical and electrical properties, stability, and biocompatibility [48]. Convergence of nanotechnology with functional molecules has led to further breakthroughs in the field of healthcare [49-51]. Diverse NPs, including quantum dots (QDs) [52], gold NPs (AuNPs) [53], and polymeric NPs [54], have been used for bioconjugation to DNAzymes.

Another technique that has been incorporated into SELEX to improve the selection of aptamers is microscopy _ for example, stimulated emission depletion microscopy (STED) and laser confocal fluorescence microscopy (LCFM) $[55,56]$. One highlight of this strategy is that it allows extraction of a single aptamer molecule. PCR amplification of these single-selected aptamers allows direct sequencing, thus eliminating several laborious steps of the SELEX procedure such as cloning and purification. This approach is still under development because it cannot be applied to a large pool of oligonucleotides. This technique offered a new method of aptamer selection in an exceptionally short period of time. These new-age microscopy techniques will be particularly helpful for aptamer libraries based on chemically modified nucleotides.

SELEX is an important tool for the selection of functional DNA molecules. Numerous investigations and research studies have been carried out to make it more efficient, quick, and selective to generate more modified versions of SELEX. Although SELEX was initially developed for the selection of DNA/RNA aptamer molecules, modification 
of the technology has allowed selection of functional nucleic acidbased catalytic molecules. File S1 (supplemental information) summarizes all SELEX methods available to date with a brief explanation of their basic principles.

\section{DNAzyme-Catalyzed Reactions}

DNAzyme-catalyzed reactions constitute a new area of biological research. Despite the fact that there are only four nucleobases, DNAzymes can facilitate a wide range of reactions (Boxes $\mathbf{2}$ and 3), including cleavage and ligation of DNA or RNA [57,58], RNA branching [59], synthesis of nucleopeptide bonds [60], phosphorylation [61], depurination, adenylation, and porphyrin metallation of DNA [6,62] (Figure 4). These reactions further helped in understanding the potential of DNA as a catalytic molecule. Moreover, DNAzyme-catalyzed reactions generally require specific cofactors, principally metal ions, for catalysis [7]. The group of Silverman [6] further extended the spectrum of DNAzymes through the development of newer catalytic molecules. For example, DNAzymes catalyzing phosphatase, phosphoserine lyase, and ester/ amide hydrolase reactions have interesting future prospects in biotechnological applications.

\section{Structural Analysis of DNAzymes}

The crystal structure of a molecule is a $3 \mathrm{D}$ atomic configuration that corresponds to the lowest state of energy. DNAzymes have been shown to have a well-defined 3D conformation [63], and their detailed structures give insights into the interaction between the DNAzyme and the substrate molecule and have been applied to the design of DNAzymes for other catalytic reactions.

DNAzymes are typically classified as metallozymes, but crystallographic studies have revealed that metal ions are generally absent from the catalytic active site, although bound metal ions were found to induce additional folding [64]. This will further influence research to develop novel cofactor-independent DNAzymes. These observations confirmed that structural and mechanistic analysis is necessary to assess the complete potential of DNAzymes. Although attempts have been made to study the crystal structures of DNAzymes, more research in this area is needed. 
Box 2. DNAzyme Promiscuity for RNA/DNA Cleavage and CarbonCarbon Bond Formation

DNAzymes that catalyze the cleavage of RNA are by far the largest class of catalytic DNA molecules. RNA cleavage invariably depends on nucleophilic attack of a phosphorous linkage by the $2^{\prime}$-hydroxyl of the adjacent ribose sugar [6]. This results in the formation of 2',3'-cyclic phosphate and a 5'-hydroxyl group at the cleavage site. The basis of catalysis appears to be similar between synthetic DNAzymes and naturally occurring RNAzymes. The very first DNAzyme, GR-5 [4], was an RNA-cleaving DNAzyme, and such DNAzymes can increase the rate of target cleavage by a factor of 1010. However, DNAzymes are generally dependent on a metal ion cofactor for their enzymatic activity. The two most common types of DNAzymes discovered since GR-5 contain are 10-23 and 8-17 motifs [7]. DNAzymes of the 8-17 type are the most thoroughly studied because they can cleave multiple types of RNA substrate. The presence of a guaninethymine (G-T) 'wobble' base pair downstream of the cleavage site is indispensable for the catalytic activity of the 8-17 DNAzyme, and replacement of the wobble pair by a Watson-Crick pair at this position abolishes the cleavage reaction. This DNAzyme sequence arrangement recurs very frequently during the selection against various targets owing to its small size. More recently, efforts have been made to develop RNA-cleaving DNAzymes that function in the absence of metal ion cofactors [127], and protonated nucleotides of DNAzymes have been found to induce catalytic reactions. Subsequently, DNAzymes using organic cofactors such as histidine for catalytic activity have also been reported [58].

The development of DNA-cleaving DNAzymes was promoted by the need for synthetic DNA-based nucleases which would have wide applications in DNA cloning. The DNAzyme 10MD5 was found to cleave the target phosphodiester linkage specifically at the sequence ATG^ $\mathrm{T}$ in the presence of metal ion cofactors $\mathrm{Mn}^{2+}$ and $\mathrm{Zn}^{2+}[7,128]$.

\section{Diels-Alder Reaction}

DNAzymes can also catalyze the formation of carbon-carbon $(C-C)$ bonds [7]. The Diels-Alder reaction involves a reaction between anthracene (a conjugated diene) and maleimide derivatives (alkene) to form 9,10-dihydroanthracene- $9,10-\alpha, \beta$-succinic anhydride, and these anthracene-imide derivatives have a wide range of applications in chemical biology. Because $\mathrm{C}-\mathrm{C}$ bond formation is a fundamental reaction of all synthetic organic chemistry, bioenzymes are attracting much interest in this field. 
Box 3. DNAzyme-Mediated Catalysis of Other Types of Biochemical Reaction

Ligation

DNAzymes can catalyze the nonnative $2{ }^{\prime}-5^{\prime}$ linkage because this does not require high-energy substrates. The mechanism of RNA ligation is to form a bond between a 2', 3'-cyclic phosphate and a 5'-hydroxyl [7]. Despite success in bridging the two fragments using a DNAzyme, there is a need for potential alternative methodologies that can lead to native $3^{\prime}-5^{\prime}$ linkages. Subsequently, numerous studies successfully demonstrated the creation of native $3^{\prime}-5^{\prime}$ phosphodiester linkages in the presence of metal ion cofactors using DNAzyme catalysis. These DNAzymes ligate with a high rate of reaction. Since then, research has focused on how to generate DNAzymes that are capable of catalyzing all possible ligation reactions.

\section{Branching}

DNAzymes have been identified catalyzing formation of a $2^{\prime}-5^{\prime}$ branched RNA by reaction between an internal 2'- hydroxyl group of one oligonucleotide and a terminal $5^{\prime}$-triphosphate group of another [6]. Examples of deoxyribozymes that can create 2',5' branched RNA sequences include 10DM24, and 7S11. The DNAzyme 7S11 branching enzyme can catalyze a reaction using the three-helix junction approach. In this approach, the DNAzyme selectively recognizes a single unpaired adenosine nucleotide in one substrate molecule, which further induces a nucleophilic reaction between the 2'-hydroxyl of adenosine and the $\alpha$-phosphate of the other RNA substrate. Deoxyribozymes can also catalyze lariat RNA (closed loop at termini) reactions. The major mechanism of such reactions is similar to the formation of a looped byproduct during splicing of nascent mRNA after transcription. In addition, DNAzymes catalyzing synthesis of branched DNA have also been isolated. These recently reported new DNAzymes have great potential in catalytic research.

\section{Nucleopeptide Linkage}

A DNAzyme has been isolated that catalyzes the formation of tyrosine-RNA nucleopeptide linkages $[6,7]$. This DNAzyme was derived by reselection from the 7S11 DNAzyme in which tyrosine replaced the branch-site ribonucleotide while maintaining the three-helix junction interface. This 7S11 variant DNAzyme employed GTP for substrate-level phosphorylation.

DNAzymes that are able to act on small-molecule substrates still need to be identified, and DNA aptamers with high affinity for a broad range of molecules are a focus of ongoing research. 


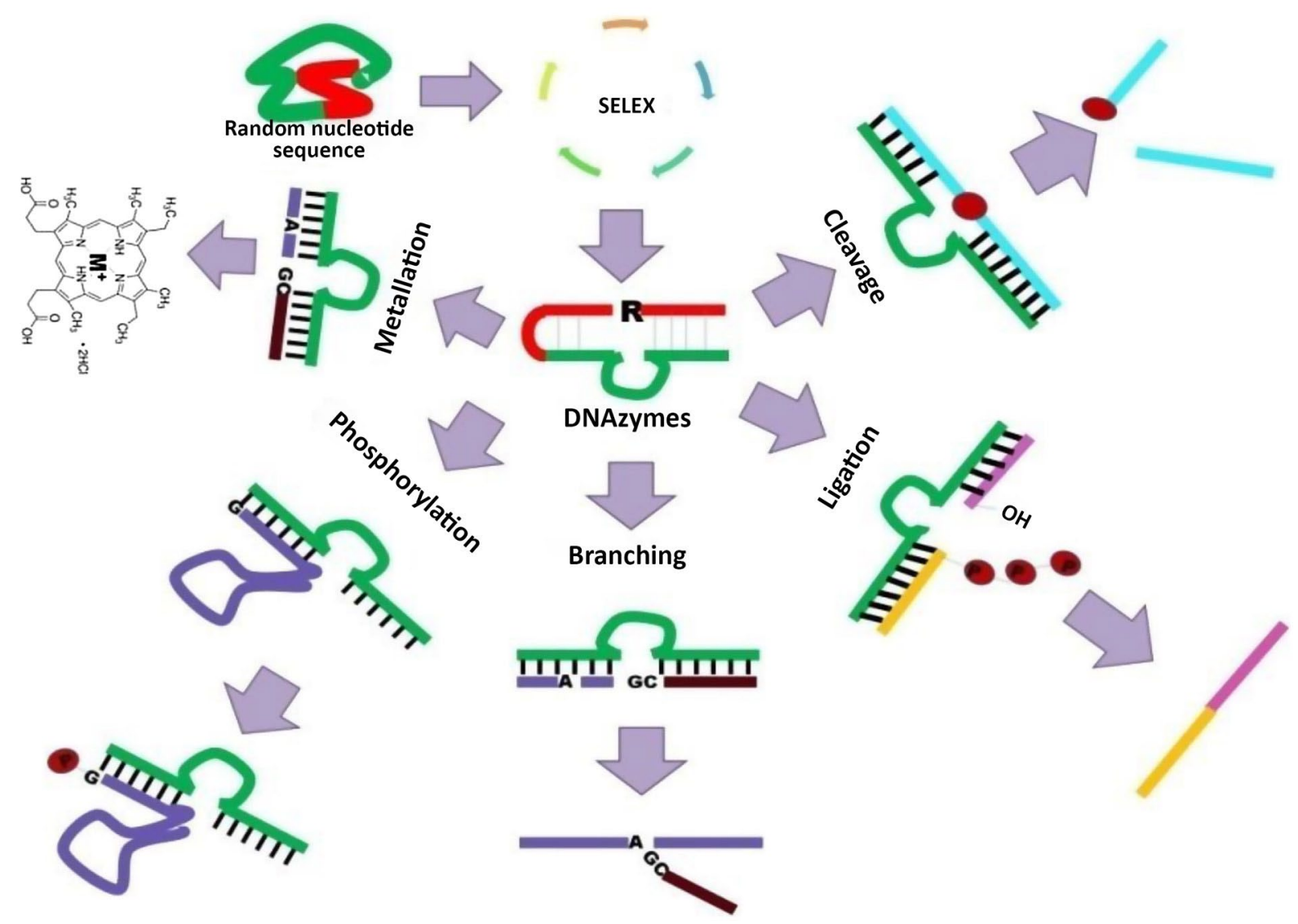

Figure 4. Schematic of Different Reactions Catalyzed by DNAzymes. Catalytic active DNA sequences are represented by green line with loop. Binding of DNAzyme can catalyze (i) cleavage, (ii) ligation, (iii) branching, (iv) phosphorylation, and (v) metallation reaction.

\section{Emerging Applications of DNAzymes}

Since the first invention of DNAzymes, these catalysts have become a hot topic of research. In addition to inherent academic interest in DNAzyme-mediated catalysis, research in this field has fostered applications across a wide range of scientific disciplines, including molecular biology tools, therapeutics, sensing, and nanomotors. Some applications are of immediate practical utility (such as the use of RNAcleaving DNAzymes as in vitro endoRNases), whereas others remain to be validated. However, in the future, innovative research might overcome additional obstacles before they can be used productively. In this section we address the potential utility of DNAzymes in various applications. 


\section{DNAzymes as Biosensors}

The development of biosensors to detect a specific analyte generally involves the integration of biological molecules with a signal transducer. Biosensors are highly dependent on the dynamics of the target molecule. Given their enhanced selectivity, stability, and high affinity (often in the picomolar to nanomolar range), DNAzymes have also been explored as biosensors. The introduction of reporter molecules into nucleic acid-based functional molecules (aptamer/ DNAzymes) can be easier than for other molecules. The first DNAzymebased fluorescent biosensor evolved from one of the most remarkable DNAzymes, 8-17, which employs metal ion $\left(\mathrm{Pb}^{2+}\right)$-dependent RNAcleavage activity for biosensing [65]. These biosensors can offer exquisite sensitivity and selectivity. Consequently, multiple types of sensors have been developed by combining DNAzymes with different signal-detection techniques (optical, piezoelectric, and electrochemical). Since then, numerous DNAzyme biosensors have been invented for the effective detection of diverse metal ions, such as $\mathrm{Cu}^{2+}, \mathrm{Pb}^{2+}$, $\mathrm{Hg}^{2+}, \mathrm{UO}_{2}{ }^{2+}$, and $\mathrm{K}^{+}$, using simple engineering methods.

\section{Optical Aptasensors}

An optical aptasensor is a device that converts light rays into an electronic signal. Optical sensing is further classified as fluorescent, colorimetric, or chemiluminescent sensing. Fluorescent sensing is among the most widely used method due to its excellent sensitivity/ selectivity, reproducibility, broad linear dynamic range of detection, and simple operational procedure. Because fluorescence is not an inherent property of DNAzyme molecules, incorporation of fluorophore molecules into the functional DNA molecules is essential to obtain fluorescence properties. DNA-based fluorescent sensors can be comprehensively characterized into labeled and nonlabeled sensors (Box 4). Nonlabeled aptasensors are generally less expensive and more straightforward to use. In addition, because there is no risk of binding-site modification, they tend to be more popular than labeled aptasensors. This approach has been applied by many groups to develop an array of label-free biosensors against a vast range of target molecules (Table 2) [66-70]. These DNAzyme-based biosensors are of great interest for real-time detection of heavy metals in water samples. 


\section{Box 4. Different Types of DNAzyme-Based Biosensors}

Nonlabeled Fluorescent Sensors

These sensors employ dyes, polymers, NPs, or nanoclusters for binding to aptamers. Intercalating dyes such as PicoGreen have been investigated for aptamer binding in sensor applications [129]. Among the different types of conjugated polymers, water-soluble polymers [e.g., poly(2,5-bis(3-sulfonatopropoxy)-1,4-phenylethynylenealt-1,4-poly(phenylene ethynylene), PPESO3] with fluorescent properties have been widely explored for use in biosensors [130], and an array of label-free biosensors have been developed against a range of target molecules.

\section{Fluorescent-Labeled Sensors}

Designing labeled aptasensors involves covalent attachment of fluorophore molecules to the target-binding sites of aptamer molecules [69]. Fluorescent nucleotide analogs have been explored in the development of aptamer sensors [131] because they are unlikely to affect binding affinity. These types of signaling aptamers can display an increment in fluorescence of up to 30-fold upon target binding. NPs can also be used instead of fluorescent dyes in the development of aptasensors. AuNPs and other metallic NP-based biosensors have been designed for a variety of targets, including cancer cells, bacterial species, and many proteins $[69,132]$. QDs take into account multiplex detection of different analytes in parallel because they can respond to similar excitation wavelengths while transmitting at different wave-lengths [71]. Graphene oxide and CNTs have attracted increasing interest in the development of DNAzyme-based biosensing frameworks because they are super-quenchers with long-range nanoscale energy-transfer properties for fluorescent assays [133].

More recently, different fluorescence modules have been developed for applications in real-time detection. The bichromophore approach is commonly used for fluorescence-based DNAzymes [68,70], and amplification methodologies such as PCR and RCA have also been investigated to enhance the detection limit of fluorescence-based aptasensors. In this respect, immuno-PCR (IPCR) has been proposed as a promising technique for the future [134].

\section{Colorimetric Aptazyme Sensors}

AuNPs and G-quadruplex aptamers have substantially contributed to the development of colorimetric biosensors because they have extremely high extinction coefficients. AuNPs also display surface plasmon resonance (SPR), an interparticle distance-dependent optical property. Cationic polymers have also been investigated for application in colorimetric aptasensors [135]. AuNPs are crosslinked to aptamers to form an aggregate, which, upon binding to the target molecule, dissociates to produce a color change [136]. Another mode of using AuNPs in colorimetric biosensing is a noncrosslinking approach. AuNPs are initially crosslinked to aptamer molecules such that they remain present in a dispersed state in a solution. Binding of the aptamer to the target molecule leads to dissociation from AuNPs, and the free AuNPs then form aggregates, leading to a color change. The rapid colorimetric aptasensor developed for $\mathrm{Pb}^{2+}$ was inspired by the same noncrosslinking approach [137]. 
Table 2. List of Different Types of DNAzyme-Based Biosensors

\begin{tabular}{|c|c|c|c|c|}
\hline Type of sensor & Biomimetic component & Reporter molecule & Analyte of interest & Reviews \\
\hline \multirow[t]{3}{*}{ Nonlabeled fluorescent sensor } & $\begin{array}{l}\text { Horseradish peroxidase } \\
\text { metallozyme-imitating } \\
\text { DNAzyme } \\
\text { (HRP-DNAzyme) }\end{array}$ & Hemin & Potassium ion $\left(\mathrm{K}^{+}\right)$ & 2012 [66] \\
\hline & DNAzyme & SYBR Green & Copper ion $\left(\mathrm{Cu}^{2+}\right)$ & $2013[67]$ \\
\hline & G quadruplex & Thioflavin T (ThT) dye & Lead ion $\left(\mathrm{Pb}^{2+}\right)$ & $2016[68]$ \\
\hline \multirow[t]{6}{*}{ Labeled fluorescent sensor } & DNAzyme & FAM & Sodium ion $\left(\mathrm{Na}^{+}\right)$ & 2015 [70] \\
\hline & DNAzyme & QDs & $\mathrm{Pb}^{2+}$ & $2011[71]$ \\
\hline & GR-5 DNAzyme & Graphene oxide & $\mathrm{Pb}^{2+}$ & $2011[133]$ \\
\hline & DNAzyme & Metal ion-modulated graphene & $\mathrm{Pb}^{2+}$ & $2011[138]$ \\
\hline & DNAzyme & Crosslinked AuNPs & $\mathrm{Pb}^{2+}$ & $2003[136]$ \\
\hline & DNAzyme & Noncrosslinked AuNPs & Adenosine & 2004 [137] \\
\hline \multirow[t]{7}{*}{ Colorimetric aptasensor } & DNAzyme & Unmodified AuNPs & $\mathrm{Pb}^{2+}$ & 2012 [139] \\
\hline & G quadruplex DNAzyme & Peptide/Au nanocomposites & ATP & 2014 [140] \\
\hline & G quadruplex DNAzyme & N-Methyl mesoporphyrin IX (NMM) & $\mathrm{Pb}^{2+}$ & $2012[74]$ \\
\hline & G quadruplex DNAzyme & Hemin & miRNA & 2017 [77] \\
\hline & G quadruplex DNAzyme & ABTS & Choline & 2018 [79] \\
\hline & DNAzyme & Hemin & ochratoxin A (OTA) & $2014[80]$ \\
\hline & DNAzyme & Graphene oxide & $\mathrm{Hg}^{2+}$ & 2015 [83] \\
\hline \multirow[t]{2}{*}{ Electrochemical sensors } & DNAzyme & Gold $(A u)$ electrode & Lead ion $\left(\mathrm{Pb}^{2+}\right)$ & $2008[88]$ \\
\hline & DNAzyme & Gold $(\mathrm{Au})$ electrode & Copper ion $\left(\mathrm{Cu}^{2+}\right)$ & 2017 [89] \\
\hline
\end{tabular}

For catalytic beacon sensors, one of the major drawbacks is background fluorescence. Such background occurs mainly due to nonspecific dehybridization of the enzyme and the target substrate. To combat these issues, immobilization of catalytic molecules has been reported, and this resulted in a $>10$-fold improvement of the detection limit. QDs were first chosen because they have greater photostability, larger Stokes shifts, and longer fluorescence periods [71]. However, NPs (e.g., iron oxide, gold, and manganese oxide) (Box 4) have been preferred for direct conjugation with DNAzymes because they have lower toxicity.

The fluorescence signals generated can be monitored using different systems such as anisotropy, bichromophores, fluorescence resonance energy transfer (FRET), and antisense con-formational triggers $[68,70]$. The detection sensitivity of DNAzyme-based point-of-care devices can be enhanced by incorporating advanced techniques such as real-time PCR and rolling circle amplification (RCA) (Box 4). Recently, reduced graphene oxide was integrated with RCA to further increase 
the versatility and sensitivity of aptazyme sensors [72]. The method includes the release of primer-tagged ATP aptamers from graphene oxide upon target binding as a result of conformational changes. These ATP aptamers were then amplified by RCA, and the limit of detection (LoD) could be improved by several orders of magnitude.

Colorimetric sensing, another type of optical sensing, detects a change in color taking place upon binding. Currently, AuNPs and Gquadruplex aptamers are most popular for the development of colorimetric aptasensors [73] (Box 4). The sensitivity of colorimetric sensors can be superior to comparable optical fluorescence sensors because they take advantage of noncovalent interactions between dyes and aptamers. The preparation of DNA-linked AuNPs is somewhat tedious, and scientists devised a new concept of unmodified AuNPs [74]. In this application, NPs were stabilized using negative charge, which prevented them from aggregation, while van der Waals interactions allowed ssDNA to coil around the AuNPs. Upon interaction with target molecules, aptamers lost their ability to protect the NPs. Hence, they formed aggregates, thus producing a color change. Likewise, AuNPs can be used in conjunction with aptamers against specific analytes [75].

G-quadruplex DNAzymes have also been tested for the development of colorimetric bio-sensing. Such complex DNAzymes can catalyze the oxidation of hydrogen peroxide that can be detected by chemiluminescence. This mechanism was employed for sensing any analyte by incorporating G-quadruplex in aptamers. The advantage of chemiluminescence as a detection unit in aptasensors is its unique zero background. The only limitation of the technique is the availability of a few chemiluminescent compounds are available. These DNAzymes have been explored for the generation of chemiluminescencebased biosensors against different targets (Table 2) [76-79].

Liu and colleagues worked on chemiluminescence resonance energy transfer (CRET)-based aptamer sensors. CRET is similar to FRET because it depends on transfer of resonance energy from chemiluminescent donors to QDs as acceptors. For CRET-based aptasensors, peroxidase-mimicking DNAzymes were conjugated to QDs [80]. The CRET principle was incorporated into many aptamers to further develop the sensing platform [81]. In addition to QDs, other nanomaterials such as graphene oxide have been employed in chemiluminescent aptasensors $[82,83]$. 


\section{Electrochemical Aptasensors}

Another type of biosensor with a wide range of applications in healthcare and environmental analysis is electrochemical detection, and electrochemical procedures with enhanced sensitivities can be easily incorporated into point-of-care devices. The instrumentation of electrochemical sensors is straightforward and easy to use. Different electrochemical strategies have been used to measure the changes in current or voltage, including impedance spectroscopy, potentiometry, electrogenerated chemiluminescence, voltammetry (cyclic/linear), and amperometry.

A major advance in the field of electrochemical biosensors is the use of nanomaterials [e.g., gold and silver NPs, multiwalled carbon nanotubes (MWCNTs), and nanoconductive polymers] to improve sensitivity, biocompatibility, and, more particularly, miniaturization [84,85]. Among them, appropriately functionalized carbon nanomaterials can be used to build superior electrochemical sensors with promising applications in diagnostics and therapeutics [86].

Electrochemical sensing strategies are further classified into two categories: labeled and label-free. The labeling methodology [87] results in high sensitivity and specificity. Electroactive compounds [e.g., ferrocene, ferrocyanide, methylene blue (MB), and QDs] were found to be more valuable labels for aptamer conjugation than were enzymes such as horseradish peroxidase, glucose oxidase, or acid phosphatase. Voltammetric pulse techniques [e.g., differential pulse voltammetry (DPV), square wave voltammetry (SWV), and alternating current voltammetry (ACV)] were most commonly used for label assays because of their high signal-to-noise ratio. The labeled methodology was further sectionalized into switch-on and switch-off types, which differ in how the electrochemical signal is generated. In the switchon approach [87], target binding induces a conformational change in the structure of the aptamer molecule. This conformational change decreases the effective distance between redox species and the electrode, leading to an increased electric current. By contrast, in the switch-off strategy [88] there is a significant separation between the electroactive compound and the electrode, prompting a reduction in the electrochemical signal. One particular example of the switch-off type is an electrochemical DNAzyme sensor for lead $\left(\mathrm{Pb}^{2+}\right)$. Following (label-free) aptamer-analyte binding, the electrochemical behavior of intrinsic redox molecules (mainly guanine and adenine) at the 
electrode surface is modified, and these redox changes can be accurately detected by electrochemical techniques such as cyclic voltammetry, potentiometry, and electrochemical impedance spectroscopy (EIS) [89]. Electrochemical aptasensors have thus become an established method for detecting analytes in clinical, environmental, and biological applications [87-91].

\section{DNAzymes as Therapeutic Agents}

The conformational plasticity and targeting specificity of these functional oligonucleotides make them a potential source of therapeutic molecules. DNAzyme-based biomedicine typically involves the modulation of downstream signaling of the desired target after binding to a functional molecule. Specifically, RNA-cleaving DNAzymes were described as promising therapeutic agents [92]. The vast majority of in vivo drug trials in the field have employed the remarkable deoxyribozyme, 10-23, because it can act upon a vast array of cleavage sites. Cancer treatment is the main therapeutic application of DNAzymes because they can distinguish between healthy and tumorigenic cells [92]. Unlike other therapeutic strategies DNAzyme-based drugs have no adverse effects on healthy cells or on the maintenance of homeostasis in the patient, DNAzymes have been used to target several key biological systems (Table $\mathbf{3}$ ), taking advantage of their specificity and stability under in vivo conditions.

Table 3. List of DNAzyme Systems Used To Treat Different Types of Cancer

\begin{tabular}{|c|c|c|c|c|}
\hline S. no. & Type of DNAzyme & Molecular target present on cancer cell & Type of cancer & Ref. \\
\hline 1 & 10-23 subclass & Early growth response protein 1 (EGR1) & Breast cancer & [141] \\
\hline 2 & DZ509 & EBV latent membrane protein & Nasopharyngeal carcinoma & [142] \\
\hline 3 & $10-23$ & Hepatitis $B$ virus $X$ protein & Hepatocellular carcinoma & [143] \\
\hline 4 & $\begin{array}{l}\text { DrzBS- and DrzBC-based } \\
\text { on the } 10-23 \text { model }\end{array}$ & Hepatitis $B$ virus $s$ and $e$ genes & Hepatocellular carcinoma & [144] \\
\hline 5 & Dz13 and As13 & C-Jun & Skin cancer & [145] \\
\hline 6 & 12-LOX & Lipoxygenase & Leukemia & [146] \\
\hline 7 & DT912 & $\mathrm{BCL}-2$ apoptotic protein & Prostate cancer & [147] \\
\hline 8 & $\begin{array}{l}\text { Peroxidase-mimicking } \\
\text { DNAzyme }\end{array}$ & Carcinoembryonic antigen (CEA) & $\begin{array}{l}\text { Oral and salivary malignant } \\
\text { tumors }\end{array}$ & [148] \\
\hline 9 & DT433 & DNA methyltransferases & Bladder cancer & [149] \\
\hline 10 & Drz2 & AKT1 (protein kinase B) & Thyroid tumor & [150] \\
\hline 11 & Drz1 and Drz2 & Surviving apoptotic protein & Breast cancer & [151] \\
\hline 12 & Drz1 and Drz2 & Insulin-like growth factor-II (IGF-II) & Hepatocarcinoma & [152] \\
\hline 13 & AM9D & MMP9 (matrix 2metalloproteinase) & Breast cancer & [153] \\
\hline 14 & DT18 & Vascular endothelial growth factor (VEGF) & Multiple myeloma & [154] \\
\hline
\end{tabular}


More extensive biomedical implementation of DNAzymes will require highly efficient and biocompatible delivery systems. Nanocomposites hold great promise $[93,94]$ because they offer many additional benefits: (i) high drug encapsulation efficiency, (ii) sustained delivery avoiding the premature release of drugs, (iii) ability to surmount different biological barriers, and (iv) specific targeting. In addition, these nanocarriers are highly biocompatible because they are nontoxic and have low immunogenicity. A growing list of NPs and their conjugates have been developed for healthcare applications, for example iron oxide NPs (IONPs), AuNPs, QDs, carbon nanotubes (CNTs), PBE functionalized graphene oxide, and $\mathrm{Fe}_{3} \mathrm{O}_{4} /$ poly(D,L-lactide) (PDLLA) nanocomposites [93-96]. The advantageous properties of DNAzymes favor their conjugation to nanocarriers for high-efficacy therapeutics [97], and several DNAzymes have been explored in the treatment of different types of cancer (Table 3).

DNAzymes have also been developed to treat viral diseases, including HIV and hepatitis infection [98,99]. DNAzymes targeting different regulatory regions of the HIV genome have been shown to inhibit the virus at different stages of the life cycle. Similarly, DNAzymes have been used for catalytic cleavage of hepatitis virus genes, thus inhibiting further virus proliferation. DNAzyme drugs have also been designed to combat antibiotic-resistant bacterial strains., and DNAzymes have been designed for specific cleavage of $\beta$-lactamase and penicillin-binding protein mRNAs in antibiotic-resistant $S$. aureus [100]. Some cardiovascular diseases [101] have also been treated with DNAzymes that aim to downregulate the expression of pathologically active genes. Specific downregulation of the expression of Egr-1 (transcription factor) can lead to decreased apoptosis and neointima (scar tissue) formation in coronary blood vessels. This in turn can contribute to increased cardiac output, neo- vascularization, and cardiomyocyte regeneration.

Although DNAzymes have numerous benefits, some limitations need to be addressed before DNAzymes can be fully exploited as therapeutic agents. The reduced reactivity of DNAzymes adversely affects the chemical functionality of the catalytic molecule [102]. The vulnerability of the catalytic sequence to nucleases also significantly affects the performance of DNAzyme molecules [103]. Therefore, further research will be necessary for the development of DNAzymebased therapeutic technologies. 


\section{DNAzyme-Based Nanodevices}

Nanodevices integrate physics, chemistry, mathematics, and biology for the nanofabrication of desired assemblies. In the life sciences, DNA and proteins are among the most promising materials for the construction of bionanodevices. In particular, nucleotides have only two principal types of pairing, A-T or $\mathrm{C}-\mathrm{G}$, and this is comparable to the binary arithmetic of computers. Hence, information in the form of base pairs and self-assembling properties allow DNA to be exploited for building nanomachines.

DNA as a digital coding system opened a new discipline related to nanobioelectronics. An important aspect of nanoelectronics research is the generation of novel materials for implementation in device fabrication [104]. DNAzymes have shown significant potential for the design and implementation of bionanodevices for biological applications. One specific clinical application of such a DNAzymebased digital circuit is as an autonomous self-monitoring treatment device. For example, the presence of excessive thrombin can cause localized bleeding leading to brain hemorrhage, but the digital release of therapeutic molecules through a triggered system may reduce the risk. Such innovative models will require more research for practical applicability.

Early emphasis was on the development of DNAzyme-based digital circuits [105]. Different logic gates are integrated to form a digital system in which DNAzymes are used as both input and output devices. In the presence of an appropriate input signal (target), DNAzymes cleave the substrate molecule which is then transformed into a fluorescence signal. In this way, fluorescence is an accurate output of the input signal. This basic computing model has been further modified for more complex logical operations. Later, Wang and coworkers constructed a DNAzyme- and silver microsphere (SMS)-based multidigit programmable DNA keypad lock system. In this system, G4-DNAzyme-hemin conjugates were immobilized on silver micro-spheres to catalyze the chromogenic oxidation of tetramethylbenzidine (TMB) by $\mathrm{H}_{2} \mathrm{O}_{2}$ [106]. This DNAzyme-based electronic device provides a good foundation for nanomedicine, and many immune-based diseases involving deregulation of microRNAs can trigger the switching device to release catalytic molecules for their degradation. 
DNAzymes can be engineered to form a lattice of a finite size, also known as DNA origami because of their self-assembly properties [107]. These DNA lattices imitate Boolean logic gates and can further be implemented to generate different computational programs. The components of the circuit are the same (i.e., two inputs and one output). The output of this circuit is a chemiluminescence signal generated from functionalized AuNPs.

Another type of DNAzyme-based nanodevice, the 'light energypowered DNA walker system', was reported by Tan and colleagues [108]. The authors demonstrated an independent but regulated DNAzyme-based walker system. In this nanorobotic device, a suitable molecular switch, such as the photosensitive moiety pyrene (aromatic hydrocarbon), is incorporated within the DNAzyme. This new approach allows precise control of the DNA walker by using light of different wavelengths and intensities. The DNA walker system consists of the RNA-cleaving 10-23 DNAzyme that moves along a template strand in the presence of metal ion cofactors. It has autonomous motion similar to that of dynein and kinesin. Research progress in this field allowed conjugation of QDs to DNAzymes [105] to construct optical devices or quadruplex DNAzymes for chemiluminescence-based nanodevices. These nanodevices can be employed in a wide range of applications [105], such as the delivery of molecular cargo, biosensing, disintegration of DNA hydrogels, and signal amplification. Very recently, a DNAzyme nanomotor was built that can detect femtomole-level thrombin, and this has great potential in clinical diagnosis [109].

\section{DNAzymes for Targeted Drug Delivery and Therapy}

DNAzymes can also be utilized as a vehicle for targeted drug delivery. The desirable properties of DNAzymes (e.g., low immunogenicity and modifiable chemical structure) make them appropriate candidates for targeted drug delivery relative to other methods such as liposomes and nanocapsulations. DNAzymes can act as stimulus-responsive caps to the pores of nanocarriers such as AuNPs and mesoporous $\mathrm{SiO}_{2} \mathrm{NPs}$ $[81,110]$. In the presence of an activating factor, DNAzyme-mediated cleavage can unlock nanocarrier pores to allow the controlled release of drugs. Allosteric DNAzymes also have potential for targeted drug delivery. A noncanonical strategy of multifunctional nanoflowers (NFs) or nanotrains can also be employed for the delivery of anticancer 
drugs [111-113]. These noncanonical (non-Watson-Crick base pairing) tunable nanostructures can be self-assembled through rollingcycle replication of duplex DNA (template and primer). The characteristic features of DNA NFs, such as nuclease resistance, exceptionally high stability, and the presence of various functional groups, are crucial for versatile biomedical applications. In addition, fluorescent tags can also be easily incorporated into the nanomotifs. Hence, NFs can be used as dual bioimaging and drug-delivery systems.

DNAzymes have also been linked to RNA interference (RNAi) systems as a vehicle for selective delivery of RNAi. The DNAzyme-RNAi conjugated system has broad clinical applications in gene regulation and drug delivery [114]. Recently, many new concepts of theranostics have evolved, and this new paradigm has been extended to DNAzymes because they have shown exponential growth in the field of therapeutics [115]. Their functional diversity and catalytic design using combinatorial selection make DNAzymes particularly interesting for theranostic applications. In addition, DNAzymes are easy to label and can be employed for real-time detection of analytes. Thus, DNAzymes hold great promise in a therapeutic paradigm combining imaging and therapy. DNAzyme theranostics remains a relatively new concept, but there are diverse potential applications of DNAzymes in this new field.

\section{Concluding Remarks and Future Perspectives}

Since their invention, DNAzymes have displayed exponential growth as potent catalysts. This is mainly due to their inherent characteristics, such as high selectivity and specificity, as discussed earlier, and because they provide a potential alternative to more traditional biocatalysts such as proteins and RNA. One of the main challenges associated with the application of DNAzymes as in vivo therapeutics is that most DNAzymes require high concentrations of divalent metal ions for maximal catalytic activity, and these concentrations are moderately higher than the anticipated concentrations in vivo. New DNAzymes requiring low in vitro metal ion concentrations [115] are likely to function more effectively under in vivo conditions.

Another limitation of DNAzyme therapeutics relates to their selective delivery into target cells because their large size and charged backbone limits cellular uptake. The in vivo performance of therapeutic 
DNAzymes is directly related to their intracellular stability. Different sets of modifications such as circularization, phosphoramidates, locked nucleic acid (LNA), 3'-3' linkages, and modified bases have been reported to increase in vivo stability [116].

Further research is needed, particularly in theranostics, and the true potential of catalytic DNAzymes has not yet been fully developed because only a small proportion of sequences have been explored. Significant progress in the DNAzyme field depends on the exploration of highly specific targets such as miRNA and chronic disease biomarkers that have practical diagnostic applications. DNAzyme-based sensitive detection of miRNA may give rise to personalized medicine for cancer patients. Likewise, future directions of DNAzyme research include the use of simulation software for combinatorial library design and in vitro selection. This will save time because such computational techniques should efficiently provide an estimate of the constructs that meet the binding affinity required for such goals.

Another emerging field related to DNA computation is the formation of DNAzyme-based programmed nanodevices. DNAzyme-based digital circuits may provide a platform for the development of more sophisticated nanodevices for medicinal and other biological applications. Major goals of future research in the context of therapeutics are in vivo stability, point-of-care devices, site-directed delivery, and monitoring devices.

\section{Outstanding Questions}

- Recent advances in SELEX have accelerated the automated generation of DNAzyme molecules, but what further improvements will be necessary?

-What are the specific characteristics of DNAzymes that allow them to be used in theranostic applications?

-What are the roles of RNA cleavage and G-quadruplexes in DNAzyme-based sensors?

-Why have the vast majority of the in vivo drug trials utilized the 10 23 DNAzyme?

- What are the major factors that affect the in vivo performance of DNAzymes? 


\section{Acknowledgments}

S.K. thanks the Department of Science and Technology (DST), the Government of India, the University of Nebraska-Lincoln (UNL), the Daugherty Water for Food Institute (DWFI), and the Indo-US Science and Technology Forum (IUSSTF) for financial support through the Water Advanced Research and Innovation (WARI) Program (award IUSSTF/WARI/2018/F-029-2018, 03-01-2018) as well as the Department of Biotechnology (DBT), Government of India (award BT/PR18868/ BCE/8/1370/2016, 31-01-2018), and DST-PURSE (Promotion of University Research and Scientific Excellence) via GJUS\&T, Hisar, under PURSE program SR/ PURSE Phase 2/40(G).

S.J. thanks the University Grants Commission (UGC), India, for providing financial assistance in the form of a Junior Research Fellowship [award F.16-6 (Dec. 2016)/2017 (NET), 12-09-2017].

K.H.K. acknowledges grant support from the National Research Foundation of Korea (NRF) funded by the MSIP (Ministry of Science, ICT, and Future Planning), Government of Korea (grant 2016R1E1A1A01940995) as well as the support made by the R\&D Center for Green Patrol Technologies through the R\&D for Global Top Environmental Technologies funded by the Ministry of Environment (MOE 2018001850001).

\section{Supplemental Information follows the References.}

\section{References}

1. Lan, T. et al. (2010) A highly selective lead sensor based on a classic lead DNAzyme. Chem. Commun. 46, 3896-3898

2. Ellington, A.D. and Szostak, J.W. (1990) In vitro selection of RNA molecules that bind specific ligands. Nature 346, 818-822

3. Tuerk, C. and Gold, L. (1990) Systematic evolution of ligands by exponential enrichment: RNA ligands to bacteriophage T4 DNA polymerase. Science 249, 505-510

4. Breaker, R.R. and Joyce, G.F. (1994) A DNA enzyme that cleaves RNA. Chem. Biol. 1, 223-229

5. Silverman, S.K. (2008) Nucleic acid enzymes (ribozymes and deoxyribozymes): in vitro selection and application. In In Wiley Encyclopedia of Chemical Biology. John Wiley \& Sons, Inc, p. wecb 406

6. Silverman, S.K. (2016) Catalytic DNA: scope, applications, and biochemistry of deoxyribozymes. Trends Biochem. Sci. 41, 595-609

7. Hollenstein, M. (2015) DNA catalysis: the chemical repertoire of DNAzymes. Molecules 20, 20777-20804

8. Willner, I. (2008) DNAzymes for sensing, nanobiotechnology and logic gate applications. Chem. Soc. Rev. 37, 1153-1165

9. Jeddi, I. and Saiz, L. (2017) Three-dimensional modeling of single stranded DNA hairpins for aptamer-based biosensors. Sci. Rep. 7, 1178 
10. Carlini, A.S. et al. (2016) Biosynthetic polymers as functional materials. Macromolecules 49, 4379-4394

11. Zhu, Q. et al. (2015) DNA aptamers in the diagnosis and treatment of human diseases. Molecules 20, 20979-20997

12. Morihiro, K. et al. (2017) Biological applications of xeno nucleic acids. Mol. Biosyst. 13, 235-245

13. Setlem, K. et al. (2016) Immuno affinity SELEX for simple, rapid, and costeffective aptamer enrichment and identification against aflatoxin B1. Front. Microbiol. 7, 1909

14. Blind, M. and Blank, M. (2015) Aptamer selection technology and recent advances. Mol. Ther. Nucleic Acids 4, e223

15. Fülle, L. et al. (2018) RNA aptamers recognizing murine CCL17 inhibit T cell chemotaxis and reduce contact hypersensitivity in vivo. Mol. Ther. 26, 95-104

16. Zhou, L. et al. (2016) G-quadruplex DNAzyme biosensor for quantitative detection of T4 polynucleotide kinase activity by using split-to-intact G-quadruplex DNAzyme conversion. Chin. J. Anal. Chem. 44, 13-18

17. Chen, Y. et al. (2018) Selection of DNA aptamers for the development of light-up biosensor to detect Pb (II). Sens. Actuators B. Chem. 254, 214-221

18. Pang, X. et al. (2018) Bioapplications of cell-SELEX-generated aptamers in cancer diagnostics, therapeutics, theranostics and biomarker discovery: a comprehensive review. Cancers 10, e47

19. Civit, L. et al. (2017) Systematic evaluation of cell-SELEX enriched aptamers binding to breast cancer cells. Biochimie 145, 53-62

20. Chen, M. et al. (2016) Development of cell-SELEX technology and its application in cancer diagnosis and therapy. Int. J. Mol. Sci. 17, 2079

21. Hung, L.Y. et al. (2015) Screening of aptamers specific to colorectal cancer cells and stem cells by utilizing on-chip cell-SELEX. Sci. Rep. 5, 10326

22. Shiratori, I. et al. (2014) Selection of DNA aptamers that bind to influenza A viruses with high affinity and broad subtype specificity. Biochem. Biophys. Res. Commun. 443, 37-41

23. Song, M.Y. et al. (2017) Broadly reactive aptamers targeting bacteria belonging to different genera using a sequential toggle cell-SELEX. Sci. Rep. 7, 43641

24. Quang, N.N. et al. (2017) Selection of aptamers against whole living cells: from cell-SELEX to identification of biomarkers. Methods Mol. Biol. 1575, 253-272

25. Raddatz, M.S. et al. (2008) Enrichment of cell-targeting and populationspecific aptamers by fluorescence-activated cell sorting. Angew. Chem. Int. Ed. Engl. 47, 5190-5193

26. Thiel, W.H. and Thiel, K.W. (2015) Cell-internalization SELEX: method for identifying cell-internalizing RNA aptamers for delivering siRNAs to target cells. Methods Mol. Biol. 1218, 187-199

27. Souza, A.G. and Marangoni, K. (2016) 3D Cell-SELEX: devel-opment of RNA aptamers as molecular probes for PC-3 tumor cell line. Exp. Cell Res. 341, $147-156$ 
28. Mi, J. et al. (2016) In vivo selection against human colorectal cancer xenografts identifies an aptamer that targets RNA helicase protein DHX9. Mol. Ther. Nucleic Acids 5, 315

29. Van Bel, N. et al. (2014) In vivo SELEX of single-stranded domains in the HIV-1 leader RNA. J. Virol. 88, 1870-1880

30. Monaco, I. et al. (2017) Aptamer functionalization of nanosystems for glioblastoma targeting through the blood-brain barrier. J. Med. Chem. 60, 4510-4516

31. Camorani, S. and Cerchia, L. (2015) Oligonucleotide aptamers for glioma targeting: an update. Cent. Nerv. Syst. Agents Med. Chem. 15, 126-137

32. Dela9c, M. et al. (2015) Aptamer for imaging and therapeutic targeting of brain tumor glioblastoma. Cytom 87, 806-816

33. Jeremy, R.B. et al. (2003) Aptamers switch on fluorescence of triphenylmethane dyes. J. Am. Chem. Soc. 125, 14716- 14717

34. Darmostuk, M. et al. (2015) Current approaches in SELEX: an update to aptamer selection technology. Biotechnol. Adv. 33, 1141-1161

35. Luo, Z. et al. (2015) Development of a fraction collection approach in capillary electrophoresis SELEX for aptamer selection. Analyst 140, 2664-2670

36. Wang, J. et al. (2014) Particle display: a quantitative screening method for generating high-affinity aptamers. Angew. Chem. Int. Ed. Engl. 126, 4896-4901

37. Gotrik, M. et al. (2018) Direct selection of fluorescence-enhancing RNA aptamers. J. Am. Chem. Soc. 140, 3583-3591

38. Lou, B. et al. (2016) The application of capillary electrophoresis for assisting whole-cell aptamers selection by characterizing complete ssDNA distribution. J. Chromatogr. 1437, 203-209

39. Dembowski, S.K. and Bowser, M.T. (2018) Microfluidic methods for aptamer selection and characterization. Analyst 143, 21-32

40. Hong, S.L. et al. (2017) Cellular-beacon-mediated counting for the ultrasensitive detection of Ebola virus on an integrated micromagnetic platform. Anal. Chem. 89, 6535-6542

41. Liu, X. et al. (2017) Selection of aptamers based on a protein microarray integrated with a microfluidic chip. Lab Chip 17, 178- 185

42. Olsen, T. et al. (2017) An integrated microfluidic SELEX approach using combined electrokinetic and hydrodynamic manipulation. SLAS Technol. 22, 63-72

43. Yüce, M. et al. (2015) Trends in aptamer selection methods and applications. Analyst 140, 5379-5399

44. Reinholt, S.J. et al. (2016) Highly multiplexed RNA aptamer selection using a microplate-based microcolumn device. Sci. Rep. 6, 29771

45. Szeto, K. et al. (2014) High-throughput binding characterization of RNA aptamer selections using a microplate-based multiplex microcolumn device. Anal. Bioanal. Chem. J. 406, 2727-2732 
46. Gopinathan, P. et al. (2017) Automated selection of aptamers against cholangiocarcinoma cells on an integrated microfluidic platform.

Biomicrofluidics 11, 044101

47. Hünniger, T. et al. (2014) Just in time-selection: a rapid semi-automated SELEX of DNA aptamers using magnetic separation and BEAMing. Anal. Chem. 86, 10940-10947

48. Liang, H. et al. (2014) Functional DNA-containing nanomaterials: cellular applications in biosensing, imaging, and targeted ther-apy. Acc. Chem. Res. 47, 1891-1901

49. Jo, H. and Ban, C. (2016) Aptamer-nanoparticle complexes as powerful diagnostic and therapeutic tools. Exp. Mol. Med. 48, e230

50. Wang, Q.Y. and Kang, Y.J. (2016) Bioprobes based on aptamer and silica fluorescent nanoparticles for bacteria Salmonella typhimurium detection. Nanoscale Res. Lett. 11, 150

51. Liu, M. et al. (2017) Aptamer selection and applications for breast cancer diagnostics and therapy. J. Nanobiotechnol. 15, 81

52. Demirkol, D.O. and Timur, S. (2016) A sandwich-type assay based on quantum dot/aptamer bioconjugates for analysis of E. coli O157: $\mathrm{H} 7$ in microtiter plate format. Int. J. Polym. Mater. Polym. Biomater. 65, 85-90

53. Belyanina, I.V. et al. (2017) In vivo cancer cells elimination guided by aptamerfunctionalized gold-coated magnetic nanoparticles and controlled with low frequency alternating magnetic field. Theranostics 7, 3326

54. Ho, L.C. et al. (2015) Aptamer-conjugated polymeric nanopar-ticles for the detection of cancer cells through 'turn-on' retro-self-quenched fluorescence. Anal. Chem. 87, 4925-4932

55. DeCastro, M.A.G. et al. (2017) Aptamers provide superior stainings of cellular receptors studied under super-resolution microscopy. PLoS One 12, e0173050

56. Long, Y. et al. (2016) Screening and identification of DNA aptamers toward Schistosoma japonicum eggs via SELEX. Sci. Rep. 6, 24986

57. Zhou, W. et al. (2015) DNAzyme hybridization, cleavage, degradation, and sensing in undiluted human blood serum. Anal. Chem. 87, 4001-4007

58. Sun, Y. et al. (2017) New cofactors and inhibitors for a DNA-cleaving DNAzyme: superoxide anion and hydrogen peroxide mediated an oxidative cleavage process. Sci. Rep. 7, 378

59. Zarnaghi, F.J. and Höbartner, C. (2013) Lanthanide cofactors accelerate DNAcatalyzed synthesis of branched RNA. J. Am. Chem. Soc. 135, 12839-12848

60. Pradeepkumar, P.I. et al. (2008) DNA-catalyzed formation of nucleopeptide linkages. Angew. Chem. Int. Ed. 47, 1753-1757

61. Camden, A.J. et al. (2016) DNA oligonucleotide 3'-phosphorylation by a DNA enzyme. Biochemistry 55, 2671-2676

62. Huang, P.J.J. et al. (2014) Ultrasensitive DNAzyme beacon for lanthanides and metal speciation. Anal. Chem. 86, 1816-1821

63. Ponce-Salvatierra, A. (2016) Crystal structure of a DNA catalyst. Nature 529, 231 
64. Liu, H. et al. (2017) Crystal structure of an RNA-cleaving DNAzyme. Nat. Commun. 8, 2006

65. Li, J. and Lu, Y. (2000) A highly sensitive and selective catalytic DNA biosensor for lead ions. J. Am. Chem. Soc. 122, 10466-10467

66. Fan, X. et al. (2012) A novel label-free fluorescent sensor for the detection of potassium ion based on DNAzyme. Talanta 89, 57-62

67. Zhang, L. et al. (2013) A label-free fluorescent molecular switch for $\mathrm{Cu}^{2+}$ based on metal ion-triggered DNA-cleaving DNAzyme and DNA intercalator. New J. Chem. 37, 1252-1257

68. Wen, Y. et al. (2016) A sensitive and label-free Pb (II) fluorescence sensor based on a DNAzyme controlled G-quadruplex/thioflavin T conformation. Sensors 16, 2155

69. Cho, E.J. et al. (2009) Applications of aptamers as sensors. Annu. Rev. Anal. Chem. 2, 241-264

70. Torabi, S.F. et al. (2015) In vitro selection of a sodium-specific DNAzyme and its application in intracellular sensing. Proc. Natl. Acad. Sci. U. S. A. 112, 5903-5908

71. Zhang, H. et al. (2011) DNAzyme-based highly sensitive electronic detection of lead via quantum dot-assembled amplification labels. Biosens. Bioelectron. $28,135-138$

72. Liu, M. et al. (2014) A graphene-based biosensing platform based on the release of DNA probes and rolling circle amplification. ACS Nano 8, 5564-5573

73. Li, S. et al. (2014) An ultrasensitive colorimetric aptasensor for ATP based on peptide/Au nanocomposites and hemin-G-quadruplex DNAzyme. RSC Adv. 4, 23185-23190

74. Miao, X.M. et al. (2012) Detection of $\mathrm{Pb}^{2+}$ at attomole levels by using dynamic light scattering and unmodified gold nanoparticles. Anal. Biochem. 421, 582-586

75. Medley, C.D. et al. (2008) Gold nanoparticle-based colorimetric assay for the direct detection of cancerous cells. Anal. Chem. 80, 1067-1072

76. Guo, L. et al. (2012) A G-quadruplex based label-free fluorescent biosensor for lead ion. Biosens. Bioelectron. 35, 123-127

77. Li, X. et al. (2017) A both-end blocked peroxidase-mimicking DNAzyme for low-background chemiluminescent sensing of miRNA. ACS Sens. 2, 810-816

78. Mahdiannasser, M. and Karami, Z. (2018) An innovative paradigm of methods in microRNAs detection: highlighting DNAzymes, the illuminators. Biosens. Bioelectron. 107, 123-144

79. Nikzad, N. and Karami, Z. (2018) Label-free colorimetric sensor for sensitive detection of choline based on DNAzyme-choline oxidase coupling. Int. J. Biol. Macromol. 115, 1241-1248

80. Mun, H. et al. (2014) Homogeneous assay of target molecules based on chemiluminescence resonance energy transfer (CRET) using DNAzyme-linked aptamers. Biosens. Bioelectron. 58, 308-313 
81. Sun, Y. et al. (2018) An ultrasensitive and ultraselective chemiluminescence aptasensor for dopamine detection based on aptamers modified magnetic mesoporous silica @ graphite oxide polymers. Sens. Actuators B Chem. 257, 312-323

82. Lin, Y. et al. (2018) A chemiluminescence biosensor for the detection of thrombin based on the aptamer composites. Spectrochim. Acta A Mol. Biomol. Spectrosc. 192, 153-158

83. Tian, Y. et al. (2015) A highly sensitive chemiluminescence sensor for detecting mercury (II) ions: a combination of exonuclease III-aided signal amplification and graphene oxide-assisted background reduction. Sci. China Chem. 58, 514-518

84. Naveen, M.H. et al. (2017) Applications of conducting polymer composites to electrochemical sensors: a review. Appl. Mater. Today 9, 419-433

85. Wang, D. et al. (2018) Electrochemical aptamer-based nanosensor fabricated on single Au nanowire electrodes for adenosine triphosphate assay. Biosens. Bioelectron. 99, 431-437

86. Yang, Y. et al. (2018) Aptamer-functionalized carbon nanomaterials electrochemical sensors for detecting cancer relevant biomolecules. Carbon 129, 380-395

87. Rapini, R. and Marrazza, G. (2017) Electrochemical aptasensors for contaminants detection in food and environment: recent advances. Bioelectrochemistry 118, 47-61

88. Shen, L. et al. (2008) Electrochemical DNAzyme sensor for lead based on amplification of DNA-Au Bio-Bar codes. Anal. Chem. 80, 6323-6328

89. Wu, J. et al. (2017) A DNAzyme-based electrochemical impedance biosensor for highly sensitive detection of $\mathrm{Cu}^{2+}$ ions in aqueous solution. Int. J. Electrochem. Sci. 12, 11666-11676

90. Yu, Z.G. and Lai, R.Y. (2018) A reagentless and reusable electrochemical aptamer-based sensor for rapid detection of ampicillin in complex samples. Talanta 176, 619-624

91. Figueroa-Miranda, G. et al. (2018) Aptamer-based electrochemical biosensor for highly sensitive and selective malaria detection with adjustable dynamic response range and reusability. Sens. Actuators B Chem. 255, 235-243

92. Fu, S. and Sun, L.Q. (2015) DNAzyme-based therapeutics for cancer treatment. Future Med. Chem. 7, 1701-1707

93. Xie, J. et al. (2010) Nanoparticle-based theranostic agents. Adv. Drug Deliv. Rev. 62, 1064-1079

94. Kumar, S. et al. (2018) Recent advances and remaining challenges for polymeric nanocomposites in healthcare applications. Prog. Polym. Sci. 80, 1-38

95. Kumar, S. et al. (2017) Carbon nanotubes: a novel material for multifaceted applications in human healthcare. Chem. Soc. Rev. 46, 158-196

96. Kumar, S. et al. (2015) Graphene, carbon nanotubes, zinc oxide and gold as elite nanomaterials for fabrication of biosensors for healthcare. Biosens. Bioelectron. 70, 498-503 
97. Farrokhi, F. et al. (2018) Delivery of DNAzyme targeting c-Myc gene using $\beta$-cyclodextrin polymer nanocarrier for therapeutic application in human breast cancer cell line. J. Drug Deliv. Sci. Technol. 80, 1-38

98. Sood, V. et al. (2007) DNA-enzyme-mediated cleavage of human immunodeficiency virus type 1 Gag RNA is significantly augmented by antisense-DNA molecules targeted to hybridize close to the cleavage site. Oligonucleotides 17, 113-121

99. Trepanier, J. et al. (2006) Cleavage of intracellular hepatitis C RNA in the virus core protein coding region by deoxyribozymes. J. Viral. Hepat. 13, 131-138

100. Hou, Z. et al. (2007) Inhibition of $\beta$-lactamase mediated oxacillin resistance in Staphylococcus aureus by a deoxyribozyme. Acta Pharmacol. Sin. 28, 1775-1782

101. Bhindi, R. et al. (2006) DNAzymes targeting the transcription factor Egr-1 reduce myocardial infarct size following ischemia-reperfusion in rats. J. Thromb. Haemost. 4, 1479-1483

102. Huang, P.J.J. and Liu, J. (2015) Rational evolution of $\mathrm{Cd}^{2+}$-specific DNAzymes with phosphorothioate modified cleavage junction and $\mathrm{Cd}^{2+}$ sensing. Nucleic Acids Res. 43, 6125-6133

103. Chakravarthy, M. et al. (2017) Novel chemically-modified DNAzyme targeting integrin alpha-4 RNA transcript as a potential molecule to reduce inflammation in multiple sclerosis. Sci. Rep. 7, 1613

104. Han, D. et al. (2012) A logical molecular circuit for programmable and autonomous regulation of protein activity using DNA aptamer-protein interactions. J. Am. Chem. Soc. 134, 20797-20804

105. Orbach, R. et al. (2015) Catalytic nucleic acids (DNAzymes) as functional units for logic gates and computing circuits: from basic principles to practical applications. Chem. Commun. 51, 4144-4160

106. Liu, X. et al. (2012) Switching photonic and electrochemical functions of a DNAzyme by DNA machines. Nano Lett. 13, 219-225

107. Zhang, C. et al. (2015) DNAzyme-based logic gate-mediated DNA selfassembly. Nano Lett. 16, 736-741

108. You, M. et al. (2012) An autonomous and controllable light-driven DNA walking device. Angew. Chemie 124, 2507-2510

109. Du, H. et al. (2018) Accelerating DNA nanomotor by branched DNAzyme for ultrasensitive optical detection of thrombin. Microchem. J. 139, 260-267

110. Zhang, Z. et al. (2013) Smart mesoporous $\mathrm{SiO}_{2}$ nanoparticles for the DNAzymeinduced multiplexed release of substrates. J. Am. Chem. Soc. 135, 1934-1940

111. Zhu, G. et al. (2013) Noncanonical self-assembly of multifunctional DNA nanoflowers for biomedical applications. J. Am. Chem. Soc. 135, 16438-16445

112. Hu, R. et al. (2014) DNA nanoflowers for multiplexed cellular imaging and traceable targeted drug delivery. Angew. Chemie 126, 5931-5936

113. Zhu, G. et al. (2013) Self-assembled, aptamer-tethered DNA nanotrains for targeted transport of molecular drugs in cancer theranostics. Proc. Natl. Acad. Sci. U.S.A. 110, 7998-8003 
114. The, Z.M. et al. (2016) A targeted DNAzyme-nanocomposite probe equipped with built-in $\mathrm{Zn}^{2+}$ arsenal for combined treatment of gene regulation and drug delivery. Sci. Rep. 6, 22737

115. Zhou, W. et al. (2017) Theranostic DNAzymes. Theranostics 7, 1010

116. Mallikaratchy, P. (2017) Evolution of complex target SELEX to identify aptamers against mammalian cell-surface antigens. Molecules 22, 215

117. Legiewicz, M. et al. (2005) Size, constant sequences, and optimal selection. RNA 11, 1701-1709

118. Sarac, I. and Meier, C. (2015) Efficient automated solid-phase synthesis of DNA and RNA 5'triphosphates. Chem. Eur. J. 21, 16421-16426

119. Chandran, S. (2017) Rapid assembly of DNA via ligase cycling reaction (LCR). Methods Mol. Biol. 1472, 105-110

120. Sack, M. et al. (2016) Express photolithographic DNA micro-array synthesis with optimized chemistry and high-efficiency photolabile groups. J. Nanobiotechnol. 14, 14

121. Antunes, D. et al. (2017) Using RNA sequence and structure for the prediction of riboswitch aptamer: a comprehensive review of available software and tools. Front. Genet. 8, 231

122. Gong, S. (2017) Computational methods for modeling aptamers and designing riboswitches. Int. J. Mol. Sci. 18, 2442

123. Lam, J.C. and Li, Y. (2010) Influence of cleavage site on global folding of an RNA cleaving DNAzyme. ChemBioChem 11, 1710-1719

124. Xing, H. et al. (2016) The effects of spacer length and composi-tion on aptamer-mediated cell-specific targeting with nanoscale PEGylated liposomal doxorubicin. ChemBioChem 17, 1111- 1117

125. Gawande, B.N. et al. (2017) Selection of DNA aptamers with two modified bases. Proc. Natl. Acad. Sci. U. S. A. 114, 2898-2903

126. Wang, J. et al. (2012) Influence of target concentration and background binding on in vitro selection of affinity reagents. PLoS On. 7, 43940

127. Kasprowicz, A. (2017) Characterization of highly efficient RNA-cleaving DNAzymes that function at acidic $\mathrm{pH}$ with no divalent metal-ion cofactors. Chem. Open 6, 46-56

128. Gu, H. et al. (2013) Small, highly active DNAs that hydrolyze DNA. J. Am. Chem. Soc. 135, 9121-9129

129. Wang, K. et al. (2015) A label-free aptasensor for highly sensitive detection of ATP and thrombin based on metal-enhanced Pico-Green fluorescence. Biosens. Bioelectron. 63, 172-177

130. Li, Y. et al. (2014) A non-labelled conjugated polymer-based fluorescence assay for the determination of adenosine triphos-phate and alkaline phosphatase. New J. Chem. 38, 4574-4579

131. Katilius, E. et al. (2006) Signaling aptamers created using fluorescent nucleotide analogs. Anal. Chem. 78, 6484-6489 
132. Wang, H.B. et al. (2013) A highly sensitive and selective biosensing strategy for the detection of $\mathrm{Pb}^{2+}$ ions based on GR-5 DNAzyme functionalized AuNPs. New J. Chem. 37, 2557-2563

133. Zhao, X.H. et al. (2011) Graphene-DNAzyme based biosensor for amplified fluorescence 'turn-on' detection of $\mathrm{Pb}^{2+}$ with a high selectivity. Anal. Chem. 83, 5062-5066

134. Fischer, N.O. (2008) Protein detection via direct enzymatic amplification of short DNA aptamers. Anal. Biochem. 373, 121-128

135. Ho, H.A. et al. (2002) Colorimetric and fluorometric detection of nucleic acids using cationic polythiophene derivatives. Angew. Chem. Int. Ed. 41, 1548-1551

136. Liu, J. and Lu, Y. (2003) A colorimetric lead biosensor using DNAzymedirected assembly of gold nanoparticles. J. Am. Chem. Soc. 125, 6642-6643

137. Liu, J. and Lu, Y. (2004) Adenosine-dependent assembly of aptazymefunctionalized gold nanoparticles and its application as a colorimetric biosensor. Anal. Chem. 76, 1627-1632

138. Wen, Y. et al. (2011) Metal ion-modulated graphene-DNAzyme interactions: design of a nanoprobe for fluorescent detection of lead (II) ions with high sensitivity, selectivity and tunable dynamic range. Chem. Commun. 47, 6278-6280

139. Miao, X.M. et al. (2012) Detection of $\mathrm{Pb}^{2+}$ at attomole levels by using dynamic light scattering and unmodified gold nanoparticles. Anal. Biochem. 421, 582-586

140. Li, S. et al. (2014) An ultrasensitive colorimetric aptasensor for ATP based on peptide/Au nanocomposites and hemin-G-quadruplex DNAzyme. RSC Adv. 4, 23185-23190

141. Mitchell, A. et al. (2004) Inhibition of human breast carcinoma proliferation, migration, chemoinvasion and solid tumour growth by DNAzymes targeting the zinc finger transcription factor EGR-1. Nucleic Acids Res. 32, 3065-3069

142. Ke, X. et al. (2011) Ebv-Imp1-targeted DNAzyme restrains nasopharyngeal carcinoma growth in a mouse C666-1 xeno-graft model. Med. Oncol. 28, 326-332

143. Hou, W. et al. (2005) In vitro cleavage of hepatitis B virus C mRNA by $10-23$ DNA enzyme. Hepatobiliary Pancreat. Dis. Int. 4, 573-576

144. Wo, J.E. et al. (2005) Effective inhibition of expression of hepatitis B virus genes by DNAzymes. World J. Gastroenterol. 11, 3504-3507

145. Cai, H. et al. (2012) DNAzyme targeting c-Jun suppresses skin cancer growth. Sci. Transl. Med. 4, 139-182

146. Liu, C. et al. (2001) Suppression of platelet-type 12-lipoxygenase activity in human erythroleukemia cells by an RNA-cleaving DNAzyme. Biochem. Biophys. Res. Commun. 284, 1077-1082

147. Yang, X. et al. (2016) Selection and antitumor activity of anti-Bcl-2 DNAzymes. Biochem. Biophys. Res. Commun. 479, 544-550 
148. Shahbazi, N. et al. (2017) A facile and rapid aptasensor based on split peroxidase DNAzyme for visual detection of carcinoembryonic antigen in saliva. Sens. Actuator B Chem. 253, 794-803

149. Wang, X. et al. (2015) Identification and characterization of DNAzymes targeting DNA methyltransferase I for suppressing bladder cancer proliferation. Biochem. Biophys. Res. Commun. 461, 329-333

150. Yang, L. et al. (2013) Akt1 inhibitory DNAzymes inhibit cell proliferation and migration of thyroid cancer cells. Asian Pac. J. Cancer Prev. 14, 2571-2575

151. Zhang, L. et al. (2012) Potential use of nucleic acid-based agents in the sensitization of nasopharyngeal carcinoma to radiotherapy. Cancer Lett. 323, $1-10$

152. Zhang, M. et al. (2013) Anti-insulin-like growth factor-IIP3 DNA-zymes inhibit cell proliferation and induce caspase-dependent apoptosis in human hepatocarcinoma cell lines. Drug Des. Dev. Ther. 7, 1089-1102

153. Hallett, M.A. et al. (2013) Anti-matrix metalloproteinase-9 DNAzyme decreases tumor growth in the MMTV-PyMT mouse model of breast cancer. Breast Cancer Res. 15, 12

154. Shen, L. et al. (2013) Antiangiogenic and antitumoral effects mediated by a vascular endothelial growth factor receptor 1 (VEGFR-1)-targeted DNAzyme. Mol. Med. 19, 377-386 